TRANSACTIONS OF THE

AMERICAN MATHEMATICAL SOCIETY

Volume 364, Number 7, July 2012, Pages 3829-3859

S 0002-9947(2012)05610-8

Article electronically published on March 1, 2012

\title{
IMPROVED BOUNDS ON METASTABILITY THRESHOLDS AND PROBABILITIES FOR GENERALIZED BOOTSTRAP PERCOLATION
}

\author{
KATHRIN BRINGMANN AND KARL MAHLBURG
}

\begin{abstract}
We generalize and improve results of Andrews, Gravner, Holroyd, Liggett, and Romik on metastability thresholds for generalized twodimensional bootstrap percolation models, and answer several of their open problems and conjectures. Specifically, we prove slow convergence and localization bounds for Holroyd, Liggett, and Romik's $k$-percolation models, and in the process provide a unified and improved treatment of existing results for bootstrap, modified bootstrap, and Froböse percolation. Furthermore, we prove improved asymptotic bounds for the generating functions of partitions without $k$-gaps, which are also related to certain infinite probability processes relevant to these percolation models.

One of our key technical probability results is also of independent interest. We prove new upper and lower bounds for the probability that a sequence of independent events with monotonically increasing probabilities contains no " $k$-gap" patterns, which interpolates the general Markov chain solution that arises in the case that all of the probabilities are equal.
\end{abstract}

\section{InTROdUCTION AND STATEMENT OF RESUlts}

In [16], Holroyd, Liggett, and Romik considered two-dimensional $k$-percolation models for $k \geq 2$. In this cellular automaton model, an initial configuration on $\mathbb{Z}^{2}$ is randomly determined by independently setting each site to be active with probability $1-q$, or empty (inactive) with probability $q$. Throughout we will write $q:=e^{-s}$. Active sites always remain active, and the system evolves by following a threshold growth rule: if $N_{k}(x)$ contains at least $k$ active sites, then $x$ becomes active, where the neighborhood is the $(k-1)$-cross given by

$$
N(x)=N_{k}(x):=\{x+w: w=(v, 0) \text { or }(0, v),-(k-1) \leq v \leq(k-1), v \neq 0\} .
$$

The case $k=2$ is the widely studied bootstrap percolation model 2, 14. Other commonly studied models include modified bootstrap percolation, as well as the Froböse model 1, 2, 14, which are frequently treated in conjunction with bootstrap percolation. Indeed, we will see throughout this paper that these latter two models can be fundamentally thought of as being associated with the "degenerate" $k=1$ case.

Received by the editors June 13, 2010 and, in revised form, February 1, 2011.

2010 Mathematics Subject Classification. Primary 05A17, 11P82, 26A06, 60C05, 60K35.

The authors thank the Mathematisches Forschungsinstitut Oberwolfach for hosting this research through the Research in Pairs Program. The first author was partially supported by NSF grant DMS-0757907 and by the Alfried Krupp Prize for Young University Teachers of the Krupp Foundation. The second author was supported by an NSF Postdoctoral Fellowship administered by the Mathematical Sciences Research Institute through its core grant DMS-0441170.

(C)2012 American Mathematical Society Reverts to public domain 28 years from publication 
One of the important questions in the study of such models is whether a given initial configuration will eventually fill the entire plane with active sites (if not, then there exist sites that will never become active). In fact, in light of Schonmann and van Enter's proofs that the critical probability for percolation on the plane is zero 17. 18, this question is more properly asked on finite square regions instead. A seminal paper by Holroyd [14] showed that there is a precise metastability threshold for bootstrap percolation, which means that the most interesting (critical) behavior occurs when the probability $q$ and the side-length $L$ satisfy an exponential relationship. The limiting threshold comes into play as $s \rightarrow 0, q \rightarrow 1$, and, simultaneously, $L \rightarrow \infty$ (the rate of exponential scaling is the critical exponent). It should also be noted that Holroyd's results built upon earlier work of Aizenman and Lebowitz 2 that proved the existence of (possibly unequal) upper and lower threshold bounds.

Subsequently, Holroyd, Liggett, and Romik also exactly described the critical exponents for each of the $k$-percolation models. These models form an infinite family of two-dimensional models that vary in the neighborhood aspect, but one can also consider percolation models with similar neighborhoods that vary in dimension. This is the subject of recent and ongoing work of Holroyd [15], and also Balogh, Bollóbas, and Morris [6, 7, who have found precise critical exponents for nearest neighbor bootstrap percolation models in all dimensions.

Such metastability thresholds can be further understood through the study of "localized" models, where all active sites must emanate from a fixed initial location (typically the origin). In particular, once the global critical exponent is known, a better understanding of localized growth can lead to refined estimates for the rate of convergence in the limit. Gravner and Holroyd used this approach to prove slow convergence estimates for bootstrap percolation in [10], and our results similarly apply to all $k$-percolation models (cf. Corollary 1.2).

In this paper we generalize Gravner and Holroyd's work to all $k$-percolation models. We define a local version of $k$-percolation that has three possible states for each cell: active, occupied, or empty. An initial configuration $\mathcal{C}$ is generated by letting the origin be active with probability $1-q$ and empty with probability $q$. All other sites are either occupied with probability $1-q$ or empty with probability $q$. Throughout we denote the corresponding probability measure by $\mathbf{P}$.

If $k>1$, then the growth rules are the following:

- An occupied site becomes active if there is at least one active site within $\ell^{1}$-distance $k$.

- An empty site $x$ becomes active if there are at least $k$ active sites in $N_{k}(x)$.

For $k=1$, there are two different models. For the modified local model we have the following rules:

- Each occupied site becomes active if there is at least one active site within $\ell^{\infty}$-distance 1.

- An empty site $x$ becomes active if there is at least one active site in each of $\{x \pm(0,1)\}$ and $\{x \pm(1,0)\}$.

For the Froböse local model we have the following rules:

- Each occupied site becomes active if there is at least one active site within $\ell^{1}$-distance 1. 
- An empty site $x$ becomes active if there are two active sites as described in the second rule of the modified case, and if the cell in the "corner" between the two sites is also active.

The results on metastability thresholds are closely related to the concept of indefinite growth in localized percolation models, which means that every site in $\mathbb{Z}^{2}$ eventually becomes active. For the remainder of this paper we focus only on this perspective. Along these lines, Gravner and Holroyd proved the following bounds for local bootstrap percolation, modified bootstrap percolation, and Froböse percolation.

Theorem (Theorem 1 in [10]). If $k=1$ (modified model) or 2, then there exist positive constants $c_{1}, c_{2}, s_{0}$ such that for $s<s_{0}$, we have

$$
\begin{aligned}
\exp \left(-2 \lambda_{k} s^{-1}+c_{1} s^{-\frac{1}{2}}\right) & \leq \mathbf{P}(\text { indefinite growth }) \\
& \leq \exp \left(-2 \lambda_{k} s^{-1}+c_{2} s^{-\frac{1}{2}}\left(\log s^{-1}\right)^{3}\right) .
\end{aligned}
$$

If $k=1$ (Froböse model), then the upper bound has the power $\left(\log s^{-1}\right)^{2}$ instead. Here the threshold constants are given as

$$
\lambda_{k}:=\frac{\pi^{2}}{3 k(k+1)} .
$$

Remark. Our notation loosely follows that of [3], and many of the results in the prior literature are stated in terms of the probability parameter $q$ rather than the exponential parameter $s$. However, all such statements are equivalent, since $q=s+O\left(s^{2}\right)$ as $s \rightarrow 0$.

Gravner and Holroyd conclude their papers on local percolation by posing several questions, which include the following problems:

- Extend the results to other bootstrap percolation models for which sharp thresholds are known to exist, including bootstrap $k$-percolation (question (iii) of [10).

- Is a power of $\log s^{-1}$ in the upper bound really necessary (question (i) of [11)?

We solve the first of these problems by proving a new, unified result for all $k \geq 1$, which includes both the modified and Froböse models in the $k=1$ case. We also make progress on the second question by showing that the power of the logarithm in the upper bound is at most $5 / 2$, which improves the power of 3 found in Gravner and Holroyd's results for $k=1$ and 2 (we reduce the power from 2 to 1 in the Froböse model).

Theorem 1.1. For each $k \geq 1$, there exists a sufficiently small $n_{0}$, and constants $c_{1}, c_{2}$ such that for $s>n_{0}$ we have

$$
\begin{aligned}
\exp \left(-2 \lambda_{k} s^{-1}+c_{1} s^{-\frac{1}{2}}\right) & \leq \mathbf{P}(\text { indefinite growth }) \\
& \leq \exp \left(-2 \lambda_{k} s^{-1}+c_{2} s^{-\frac{1}{2}}\left(\log s^{-1}\right)^{\frac{5}{2}}\right) .
\end{aligned}
$$

For the $k=1$ Froböse model, the upper bound has the power $\log s^{-1}$ instead.

Remark. Throughout the later sections of this paper we use lowercase $c$ 's to represent various (effective) constants without distinguishing them by different indices. 
Although this is a slight abuse of notation, there are a finite number of such constants in use, and one can simply adopt the supremum/infimum of the collections as necessary.

While a preliminary version of this article was under review, Gravner, Holroyd, and Morris subsequently released a manuscript that further explained the usefulness of such local bounds in the study of metastability thresholds for bootstrap percolation models. Let $p_{c}(L, k)$ denote the critical probability of the $k$-percolation model on a square lattice of side-length $L$ (see 12 for a precise definition). Following the arguments in [11, it is straightforward to show that the lower bound of Theorem 1.1 implies the following upper bounds. The basic idea is to show that the bound for the local bootstrap percolation model provides a "seed" that is sufficient to populate the entire square (the cases $k=1$ and 2 of the following result were shown by Gravner and Holroyd).

Corollary 1.2. For each $k \geq 1$, there exists $c>0$ such that as $L \rightarrow \infty$,

$$
p_{c}(L, k) \leq \frac{\lambda_{k}}{\log L}-\frac{c}{(\log L)^{3 / 2}} .
$$

Remark. The new ideas in 12 also provide a companion upper bound for the critical probability. Taken together, these two bounds (nearly) specify the lowerorder asymptotic behavior of the critical probabilities; in particular, as $L \rightarrow \infty$ we have

$$
p_{c}(L, k)=\frac{\lambda_{k}}{\log L}-\frac{1}{(\log L)^{3 / 2+o(1)}} .
$$

There are two main components underlying our improvements. First, the combinatorial arguments used in Gravner and Holroyd's upper bound results for $k=1$ and 2 do not immediately generalize to higher $k$, and we adopt a significantly different conceptual approach in order to unify all cases. Much of the literature in this subject, including [2, 14, 10, 11, 16, uses the concept of internally spanned rectangles when studying upper bounds; the general idea being that if a large rectangle eventually becomes active, then there must be a nested subsequence of smaller rectangles that each became active during the overall growth process.

Our new approach comes from the observation that in their final form, these previous arguments replace internally spanned rectangles by rectangles that satisfy certain row and column conditions. In other words, the combinatorial approach is unnecessarily "tighter" than the bounds that are actually proven, and the arguments are therefore more restrictive (and thus more complicated) than is necessary. We introduce a new combinatorial construction that is more precisely tailored to reflect the row and column conditions that are actually used in approximations. The combinatorial approach used in the proof of the $k=1$ and $k=2$ cases of the lower bound of Theorem 1.1 then generalize in a more straightforward manner.

The second new component of our arguments is a two-sided bound for the probabilities of certain pattern-avoiding sequences that naturally underlie the preceding applications to bootstrap percolation models (a very rough approximation of these bounds was proven and used in [16]). For $s \in(0,1)$, let $C_{1}, \ldots, C_{n}, \ldots$ be independent events with probabilities

$$
\mathbf{P}\left(C_{n}\right)=1-e^{-n s} .
$$


Let $A_{k}$ be the event that there are no $k$-gaps among the occurrences of the events $C_{i}$, which means that there are no consecutive $C_{i}$ 's that do not occur. Symbolically, this can be written as

$$
A_{k}:=\bigcap_{i=1}^{\infty}\left(C_{i} \cup C_{i+1} \cup \ldots \cup C_{i+k-1}\right) .
$$

Holroyd, Liggett, and Romik described the logarithmic limiting behavior of these probabilities.

Theorem (Theorem 2 in [16]). For every $k \geq 1$,

$$
-\log \mathbf{P}\left(A_{k}\right) \sim \lambda_{k} s^{-1} \quad \text { as } s \rightarrow 0 .
$$

Remark. Although this is not stated in the paper, equation (14) from Section 3 of [16] immediately implies that if $s$ is sufficiently small, then there exists a positive constant $c_{2}$ such that

$$
-\log \mathbf{P}\left(A_{k}\right) \leq \lambda_{k} s^{-1}+c_{2} s^{-\frac{1}{2}} \log s^{-1} .
$$

Equation (14) in [16] also leads to a corresponding implicit lower bound, although an additional technical result is needed. Using Lemma 3.1 part (ii) from later in the present paper, the resulting bound would state that there is a positive constant $c_{1}$ such that

$$
\lambda_{k} s^{-1}-c_{1} s^{-\frac{1}{2}} \log s^{-1} \leq-\log \mathbf{P}\left(A_{k}\right) .
$$

The probability events $A_{k}$ are also of interest in combinatorial number theory and the theory of partitions, and Holroyd, Ligget, and Romik thoroughly explained the connections in [16. They showed that

$$
G_{k}(q)=\sum_{n \geq 0} p_{k}(n) q^{n}=\frac{\mathbf{P}\left(A_{k}\right)}{(q ; q)_{\infty}}=\mathbf{P}\left(A_{k}\right) \cdot \sum_{n \geq 1} p(n) q^{n},
$$

where $p(n)$ denotes the number of integer partitions of $n$, and $p_{k}(n)$ denotes the number of partitions of $n$ without $k$-sequences, i.e., those partitions that do not contain any $k$ consecutive integers as parts. We have also used the standard notation $(a ; q)_{n}=(a)_{n}:=\prod_{j=1}^{n-1}\left(1-a q^{j}\right)$ for the rising $q$-factorial.

These partitions were further studied by Andrews in [3], who found the explicit (double hypergeometric) $q$-series expansion

$$
G_{k}(q)=\frac{1}{(q ; q)_{\infty}} \sum_{r, s \geq 0} \frac{(-1)^{s} q^{\left(\begin{array}{c}
k+1 \\
2
\end{array}\right)(s+r)^{2}+(k+1)\left(\begin{array}{c}
+2+1 \\
2
\end{array}\right)}}{\left(q^{k} ; q^{k}\right)_{s}\left(q^{k+1} ; q^{k+1}\right)_{r}} .
$$

In the case $k=2$, he also found the alternative expression

$$
G_{2}(q)=\frac{\left(-q^{3} ; q^{3}\right)_{\infty}}{\left(q^{2} ; q^{2}\right)_{\infty}} \cdot \chi(q)
$$

where

$$
\chi(q):=1+\sum_{n \geq 1} \frac{q^{n^{2}}}{\prod_{j=1}^{n}\left(1-q^{j}+q^{2 j}\right)}
$$


is one of Ramanujan's third-order mock theta functions [19]. He then used the cuspidal expansions of mock theta functions and modular forms to prove the exact (non-logarithmic) asymptotic formula in the case $k=2$ :

$$
\mathbf{P}\left(A_{2}\right) \sim \sqrt{\frac{\pi}{2}} s^{-\frac{1}{2}} \exp \left(-\lambda_{2} s^{-1}\right) \quad \text { as } s \rightarrow 0 .
$$

Remark. The present authors showed in [8] that although this series asymptotically corresponds to the "dominant cusp" for $G_{2}(q)$, it does not provide sufficient information to determine the full asymptotic expansion of the $q$-series coefficients $p_{2}(n)$, or even the entire leading exponential term. The authors developed and used an amplified version of the Hardy-Ramanujan circle method in order to find formulas for the coefficients of such functions (general products of mock theta functions and modular forms), with error at most $O(\log n)$.

Although the functions $G_{k}(q)$ do not seem to be automorphic forms for $k \geq 3$, Andrews also found numerical evidence for the asymptotic in the general case and identified theta function components that make asymptotically "large" contributions. This led him to a conjectural formula for the general asymptotic.

Conjecture (Andrews [3]). For each $k \geq 2$, there is a positive constant $c_{k}$ such that

$$
\mathbf{P}\left(A_{k}\right) \sim c_{k} s^{-\frac{1}{2}} \exp \left(-\lambda_{k} s^{-1}\right) \quad \text { as } s \rightarrow 0 .
$$

We greatly refine Holroyd, Liggett, and Romik's theorem for the logarithmic asymptotic of $\mathbf{P}\left(A_{k}\right)$ (as well as the implicit bounds (1.3) and (1.4)), and also make significant progress toward Andrews' conjecture by proving (non-logarithmic) lower and upper bounds that asymptotically differ by less than a multiplicative factor of $s$.

Theorem 1.3. For every positive integer $k$, we have the following asymptotic as $s \rightarrow 0$ :

$$
\exp \left(-\lambda_{k} s^{-1}\right) \leq \mathbf{P}\left(A_{k}\right) \leq s^{-\frac{(2 k-1)}{2 k}(1+o(1))} \exp \left(-\lambda_{k} s^{-1}\right) .
$$

Remark. In fact, our proof shows that $\mathbf{P}\left(A_{k}\right)$ lies between lower and upper bounds that differ by a multiplicative factor of at most $s^{-(k-1) / k}$. However, in order to write both bounds in a clean manner, we have expanded the range to $s^{-(2 k-1) / 2 k}$ in the theorem statement.

One very interesting feature of this result is that we have used arguments from combinatorics and probability in order to conclude better asymptotic bounds for the coefficients of a $q$-series. In the subject of combinatorial $q$-series, such implications more frequently proceed in the opposite direction. For example, Andrews' work on the $k=2$ case used analytic and automorphic properties of modular forms and mock theta functions in order to conclude more precise combinatorial and probabilistic statements 3,8 .

The remainder of the paper is organized as follows. In Section 2 we define a class of certain pattern-avoiding probability sequences and prove an important new general bound for the probability of sequences without $k$-gaps. In Section 3 we turn to a logarithmic version of the function from Section 2 and prove several useful estimates that are used in bounds throughout the rest of the paper. The proof of Theorem 1.1 is found in the next two sections, and is split into the lower and upper bounds. Section 4 contains the proof of the lower bound in Theorem 
1.1. which combines generalized versions of the combinatorial ideas from [11] with the estimates from Section 3 The upper bound then follows in Section 5 In order to adapt Gravner and Holroyd's arguments in [10] to the case of general $k$, we introduce a conceptually different combinatorial model for tracking the possible ways a configuration can grow. We also make slight (and optimal) modifications to some of the parameters used in Gravner and Holroyd's arguments in order to achieve an improved $\log s^{-1}$-power. Finally, Theorem 1.3 is proved in Section 6 by adapting the general probability results of Section 2 to the specific cases described in the theorem statement.

\section{Probability Results}

2.1. Definitions and notation. In [16, Holroyd, Liggett, and Romik introduced and studied an interesting family of functions. For each positive integer $k, f_{k}(x)$ is defined to be the unique, decreasing function on $[0,1]$ that satisfies the functional equation

$$
f^{k}(x)-f^{k+1}(x)=x^{k}-x^{k+1} .
$$

Remark. Note that $f_{k}(0)=1$ and $f_{k}(1)=0$ for all $k$, and also that the first two cases have simple explicit equations; namely, $f_{1}(x)=1-x$ and $f_{2}(x)=\frac{1-x+\sqrt{(1-x)(1+3 x)}}{2}$ (this case was studied extensively by Holroyd in [14]).

As was pointed out in [16, the existence and (Lipschitz) continuity of $f_{k}$ follows immediately from the fact that $h_{k}(x):=x^{k}-x^{k+1}$ is strictly increasing for $0 \leq x<$ $\frac{k}{k+1}$ and is strictly decreasing for $\frac{k}{k+1}<x \leq 1$. We will also require the following equivalent "long" form of (2.1):

$$
f^{k}=(1-x)\left(f^{k-1}+x f^{k-2}+\cdots+x^{k-1}\right) .
$$

The main result in this section is a nearly tight probability bound that is used in many places throughout the paper. A sequence of probabilistic events is said to have a $k$-gap if there are $k$ consecutive events that do not occur.

Theorem 2.1. Suppose that $A_{1}, \ldots, A_{n}$ are independent events that each occur with probability $\mathbf{P}\left(A_{i}\right)=u_{i}$, where $u_{1}, \ldots, u_{n}$ form a monotonically increasing sequence. Then this sequence of events satisfies

$$
\prod_{i=1}^{n} f_{k}\left(1-u_{i}\right) \leq \mathbf{P}\left(\left\{A_{i}\right\}_{i=1}^{n} \text { has no k-gaps }\right) \leq \prod_{i=k}^{n} f_{k}\left(1-u_{i}\right) .
$$

Remark. If $n<k$, then the empty product on the right-hand side should be interpreted as having the value 1.

Remark. If we take the limit $n \rightarrow \infty$, then the case of monotonically increasing probabilities $u_{i}$ is essentially the only interesting possibility. If the probabilities oscillate indefinitely, then all of the terms in Theorem 2.1 approach zero rapidly.

The proof of both bounds will use inductive arguments. Define the shorthand notation

$$
\rho_{n}:=\mathbf{P}\left(\left\{A_{i}\right\}_{i=1}^{n} \text { has no } k \text {-gaps }\right),
$$

and observe that the initial $k$ values are clearly $\rho_{0}=\ldots=\rho_{k-1}=1$, all of which satisfy the theorem bounds. 
The inductive argument for all other cases follows from a simple combinatorial recurrence. Observe that if the event $\rho_{n+k}$ occurs (where $n \geq 0$ ), then at least one of $A_{n+1}, \ldots, A_{n+k}$ must occur. Separating into distinct cases based on the final such $A_{n+i}$ leads to the following recurrence:

$\rho_{n+k}=\rho_{n+k-1} u_{n+k}+\rho_{n+k-2} u_{n+k-1}\left(1-u_{n+k}\right)+\ldots+\rho_{n} u_{n+1}\left(1-u_{n+2}\right) \ldots\left(1-u_{n+k}\right)$.

The first term corresponds to the case where $A_{n+k}$ occurs, the second term the case where $A_{n+k-1}$ occurs and $A_{n+k}$ does not, and so on until the final term, which represents the case where only $A_{n+1}$ occurs.

Remark. If $k=1$, Theorem 2.1 is actually an equality (which is seen to be trivially true upon recalling that $\left.f_{1}(x)=1-x\right)$. Gravner and Holroyd proved the lower bound for the case $k=2$ in [11] by using the explicit formula for $f_{2}(x)$. Furthermore, Holroyd, Liggett, and Romik [16] also proved the theorem for general $k$ in the very special case that all $u_{i}$ are equal. Their argument essentially treats (2.3) as a linear recurrence and shows that $f_{k}$ gives the largest eigenvalue. Alternatively, this is equivalent to calculating the limiting entropy of the corresponding Markov process as $n$ increases.

2.2. Lower bound for probabilistic $k$-gaps. We prove a result that is more general than the lower bound in Theorem 2.1, as it also allows for decreasing probabilities.

Proposition 2.2. Suppose that $A_{1}, \ldots, A_{n}$ are independent events whose corresponding probabilities $u_{1}, \ldots, u_{n}$ are either increasing or decreasing. Then this sequence of events satisfies

$$
\mathbf{P}\left(\left\{A_{i}\right\}_{i=1}^{n} \text { has no k-gaps }\right) \geq \prod_{i=1}^{n} f_{k}\left(1-u_{i}\right) .
$$

This proposition will follow from an inductive argument that relies on an auxiliary function that is essentially an approximation of (2.3). For $k \geq 1$, let

$$
\begin{aligned}
H_{k}\left(y_{1}, \ldots, y_{k}\right) & :=\left(1-y_{k}\right) f_{k}\left(y_{1}\right) \ldots f_{k}\left(y_{k-1}\right)+\left(1-y_{k-1}\right) y_{k} f_{k}\left(y_{1}\right) \ldots f_{k}\left(y_{k-2}\right) \\
& +\ldots+\left(1-y_{i}\right) y_{i+1} \ldots y_{k} f_{k}\left(y_{1}\right) \ldots f\left(y_{i-1}\right) \\
& +\ldots+\left(1-y_{1}\right) y_{2} \ldots y_{k}-f_{k}\left(y_{1}\right) \ldots f_{k}\left(y_{k}\right) .
\end{aligned}
$$

Note that $H_{k}(y, \ldots, y)=0$ by (2.2). The most important property of this function is found in the following proposition.

Proposition 2.3. For increasing arguments $0 \leq y_{1} \leq \ldots \leq y_{k} \leq 1$, the function $H_{k}\left(y_{1}, \ldots, y_{k}\right)$ is non-negative.

We further postpone the proof of Proposition 2.3 until after we have proven several necessary intermediate results.

Lemma 2.4. Consider the domain $0 \leq y \leq 1$.

(i) The function $\frac{y}{f_{k}(y)}$ is increasing.

(ii) We have

$$
\frac{f_{k}^{\prime}(y)}{f_{k}(y)} \geq-\frac{1}{1-y}
$$


Proof. (i) This holds because the functions $y$ and $\frac{1}{f_{k}(y)}$ are both increasing and non-negative.

(ii) Differentiating (2.2), we find that

$$
\begin{aligned}
& f_{k}^{\prime}\left(k f_{k}^{k-1}-(1-y)(k-1) f_{k}^{k-2}-\ldots-(1-y) y^{k-2}\right) \\
& \quad=-f_{k}^{k-1}+(1-2 y) f_{k}^{k-2}+\ldots+\left((k-1) y^{k-2}-k y^{k-1}\right)
\end{aligned}
$$

$\stackrel{2.2}{\rightleftharpoons} \frac{f_{k}^{\prime}}{f_{k}}\left((1-y) f_{k}^{k-1}+2(1-y) y f_{k}^{k-2}+\ldots+(k-1)(1-y) y^{k-2} f_{k}+k(1-y) y^{k-1}\right)$

$$
=-f_{k}^{k-1}+(1-2 y) f_{k}^{k-2}+\ldots+\left((k-1) y^{k-2}-k y^{k-1}\right)
$$

$\Longleftrightarrow \frac{f_{k}^{\prime}}{f_{k}}=-\frac{1}{1-y}+\frac{f_{k}^{k-2}+2 y f_{k}^{k-3}+\ldots+(k-1) y^{k-2}}{(1-y)\left(f_{k}^{k-1}+2 y f_{k}^{k-2}+\ldots+k y^{k-1}\right)}$.

This easily gives the claim.

Next, for $1 \leq j \leq k$ define

$$
T_{k, j}(y)=T_{j}:=\frac{(1-y) y^{j-1}}{f_{k}^{j}(y)} .
$$

This family of functions interpolates between $T_{1}$ and $T_{k}$, whose opposing behaviors are described in the next result.

Lemma 2.5. The following hold for every $k \geq 1$ :

(i) The function $T_{1}$ is decreasing.

(ii) The function $T_{k}$ is increasing.

Proof. $\quad(i)$ We differentiate (2.5) to get

$$
T_{1}^{\prime}=\frac{1}{f_{k}}\left(-1+(1-y)\left(\frac{-f_{k}^{\prime}}{f_{k}}\right)\right),
$$

and use Lemma 2.4 (ii) to conclude that this is non-positive.

(ii) We have

$$
T_{k}^{\prime}=\frac{(k-1) y^{k-2}-k y^{k-1}}{f_{k}^{k}}-\frac{k f_{k}^{\prime}\left(y^{k-1}-y^{k}\right)}{f_{k}^{k+1}} .
$$

Thus $T_{k}^{\prime} \geq 0$ is equivalent to

$$
-\frac{k f_{k}^{\prime}\left(y-y^{2}\right)}{f_{k}} \geq-(k-1)+k y \Longleftrightarrow \frac{-f_{k}^{\prime}}{f_{k}} \geq \frac{k y-(k-1)}{k y(1-y)} .
$$

Inserting (2.4) implies that (2.6) is again equivalent to

$\frac{1}{1-y}-\frac{f_{k}^{k-2}+2 y f_{k}^{k-3}+\ldots+(k-1) y^{k-2}}{(1-y)\left(f_{k}^{k-1}+2 y f_{k}^{k-2}+\ldots+k y^{k-1}\right)} \geq \frac{k y-(k-1)}{k y(1-y)}$,

which further simplifies to

$$
\begin{aligned}
(k-1) f_{k}^{k-1}+2(k-1) y f_{k}^{k-2}+\ldots & +(k-1) k y^{k-1} \\
& \geq k y f_{k}^{k-2}+2 k y^{2} f_{k}^{k-3}+\ldots+k(k-1) y^{k-1} .
\end{aligned}
$$

Comparing like powers of $f_{k}$ directly shows that this last inequality is satisfied for $k \geq 2$ (observe that for $k=1$ the Lemma's claim is trivial). 
Remark. Interestingly, it can also be shown that for any $2 \leq j \leq k-1, T_{j}$ is increasing on some interval $\left[0, \sigma_{j}\right]$, and is then decreasing on $\left[\sigma_{j}, 1\right]$. We do not devote any space to proving this, as it is not needed in the sequel.

We next define the accumulation functions for $1 \leq j \leq k$ as

$$
D_{k, j}(y)=D_{j}(y):=T_{1}(y)+\ldots+T_{j}(y) .
$$

Note that by (2.2) we have the identity $D_{k}(y)=1$. Although the individual components $T_{j}$ have varying behavior, their sums are much more uniform.

Lemma 2.6. The functions $D_{j}$ are decreasing for all $1 \leq j \leq k$.

Proof. We proceed inductively and note that $D_{k}=1$ is decreasing. Now suppose that $D_{j}$ is decreasing. Then

$$
D_{j-1}^{\prime}=T_{1}^{\prime}+\ldots+T_{j-1}^{\prime}
$$

If $T_{i}^{\prime}(y) \leq 0$ for all $1 \leq i \leq j-1$ and all $y \in[0,1]$, then $D_{j-1}$ is clearly decreasing. Otherwise, if there is some $y$ and some $1 \leq i \leq j-1$ such that $T_{i}^{\prime}(y)>0$, then we also have that

$$
T_{j}^{\prime}(y)>0,
$$

since $T_{j}=T_{i}\left(\frac{y}{f_{k}}\right)^{j-i}$, and both factors are non-negative and increasing at $y$. Then

$$
D_{j-1}^{\prime}(y)=D_{j}^{\prime}(y)-T_{j}^{\prime}(y) \leq D_{j}^{\prime}(y) \leq 0
$$

by the induction hypothesis.

Proof of Proposition 2.3. We factor out the product of all of the $f_{k}\left(y_{i}\right)$ from the definition of $H_{k}$ and write

$$
\begin{aligned}
H_{k}\left(y_{1}, \ldots, y_{k}\right) & =f_{k}\left(y_{1}\right) \ldots f_{k}\left(y_{k}\right)\left(\frac{1-y_{k}}{f_{k}\left(y_{k}\right)}+\frac{\left(1-y_{k-1}\right) y_{k}}{f_{k}\left(y_{k-1}\right) f_{k}\left(y_{k}\right)}+\ldots\right. \\
& \left.+\frac{\left(1-y_{i}\right) y_{i+1} \ldots y_{k}}{f_{k}\left(y_{i}\right) f_{k}\left(y_{i+1}\right) \ldots f_{k}\left(y_{k}\right)}+\ldots+\frac{\left(1-y_{1}\right) y_{2} \ldots y_{k}}{f_{k}\left(y_{1}\right) \ldots f_{k}\left(y_{k}\right)}-1\right) .
\end{aligned}
$$

Consider the second-to-last term, which can be written as

$$
\frac{y_{2} \ldots y_{k}}{f_{k}\left(y_{2}\right) \ldots f_{k}\left(y_{k}\right)} \cdot D_{1}\left(y_{1}\right) \text {. }
$$

Lemma 2.6 states that $D_{1}\left(y_{1}\right) \geq D_{1}\left(y_{2}\right)$, so we can replace $y_{1}$ by $y_{2}$ without increasing (2.8). After this substitution, there are now two terms that contain $y_{2}$, and the combination of them can be written as

$$
\frac{y_{3} \ldots y_{k}}{f_{k}\left(y_{3}\right) \ldots f_{k}\left(y_{k}\right)} \cdot D_{2}\left(y_{2}\right) \text {. }
$$

Again we can use Lemma 2.6 and replace $y_{2}$ by $y_{3}$ without increasing the result. Continuing in this way, we successively increase the indices of all of the $y_{i}$ 's until (2.8) becomes

$$
H_{k}\left(y_{1}, \ldots, y_{k}\right) \geq f_{k}\left(y_{1}\right) \ldots f_{k}\left(y_{k}\right)\left(D_{k}\left(y_{k}\right)-1\right)=0 .
$$


Proof of Proposition 2.2. As mentioned earlier, we use an inductive argument based on (2.3). Without loss of generality, assume that the $u_{i}$ are decreasing (if they are increasing, simply reverse the order) and recall that the proposition holds for the initial cases.

The inductive hypothesis implies that every term on the right-hand side of (2.3) has a lower bound in terms of the $f_{k}$, which gives

$$
\begin{aligned}
\rho_{n+k} & \geq \prod_{i=1}^{n} f_{k}\left(1-u_{i}\right)\left(u_{n+k} f_{k}\left(1-u_{n+1}\right) \ldots f_{k}\left(1-u_{n+k-1}\right)\right. \\
& +u_{n+k-1}\left(1-u_{n+k}\right) f_{k}\left(1-u_{n+1}\right) \ldots f_{k}\left(1-u_{n+k-2}\right) \\
& \left.+\ldots+u_{n+1}\left(1-u_{n+2}\right) \ldots\left(1-u_{n+k}\right)\right) \\
& =\prod_{i=1}^{n} f_{k}\left(1-u_{i}\right)\left(H_{k}\left(y_{n+1}, \ldots, y_{n+k}\right)+f_{k}\left(1-u_{n+1}\right) \ldots f_{k}\left(1-u_{n+k}\right)\right),
\end{aligned}
$$

where $y_{i}:=1-u_{i}$, so the $y_{i}$ are increasing. Then by Proposition 2.3 we have the required bound

$$
\rho_{n+k} \geq \prod_{i=1}^{n+k} f_{k}\left(1-u_{i}\right) .
$$

Remark. Numerical evidence (and the naive heuristic of grouping together the events with larger probabilities) suggests that Proposition 2.2 may also hold for arbitrary probabilities $u_{i}$, and that the cases where the $u_{i}$ are monotonically decreasing or increasing (or more generally, unimodular) give the tightest bounds.

2.3. Upper bound for $k$-gaps. We also prove the upper bound of Theorem 2.1 as a separate statement for easier reference.

Proposition 2.7. Let $A_{1}, \ldots, A_{n}$ be independent events with probabilities $1 \geq u_{1} \geq$ $u_{2} \geq \ldots \geq u_{n} \geq 0$. Then

$$
\mathbf{P}\left(\left\{A_{i}\right\}_{i=1}^{n} \text { has no k-gaps }\right) \leq \prod_{i=1}^{n-k+1} f_{k}\left(1-u_{i}\right) .
$$

As before, we will use an auxiliary function, although in order to prove an upper bound, the function needs to have bounds that are opposite from those proven for $H_{k}$. Let

$$
\begin{aligned}
\widetilde{H}_{k}\left(y_{1}, y_{2}, \ldots, y_{2 k-1}\right) & :=\left(1-y_{2 k-1}\right) f_{k}\left(y_{1}\right) \ldots f_{k}\left(y_{k-1}\right) \\
& +\left(1-y_{2 k-2}\right) y_{2 k-1} f_{k}\left(y_{1}\right) \ldots f_{k}\left(y_{k-2}\right)+\ldots \\
& +\left(1-y_{k+i}\right) y_{k+i+1} \cdots y_{2 k-1} f_{k}\left(y_{1}\right) \ldots f_{k}\left(y_{i}\right)+\ldots \\
& +\left(1-y_{k}\right) y_{k+1} \ldots y_{2 k-1}-f_{k}\left(y_{1}\right) \ldots f_{k}\left(y_{k}\right),
\end{aligned}
$$

and note that this function has "decoupled" the variables that appear in polynomial terms from those that appear as the arguments of $f_{k}$. In particular, $\widetilde{H}_{k}$ is a linear function in each of $y_{k+1}, \ldots, y_{2 k-1}$.

Proposition 2.8. For $0 \leq y_{1} \leq \ldots \leq y_{2 k-1} \leq 1$, we have that $\widetilde{H}_{k}\left(y_{1}, \ldots, y_{2 k-1}\right) \leq$ 0 . 
Proof. As in the proof of Proposition 2.2. we will eventually change all of the $y_{i}$ 's to $y_{k}$ by using properties of the functions $D_{j}(y)$, but this time we will also need to study the partial derivatives of the linear terms of $\widetilde{H}_{k}$ in order to do so.

For the first step, consider the coefficient of $y_{k+1}$ in (2.9), which is

$-y_{k+2} \ldots y_{2 k-1} f_{k}\left(y_{1}\right)+\left(1-y_{k}\right) y_{k+2} \ldots y_{2 k-1}=y_{k+2} \ldots y_{2 k-1} f_{k}\left(y_{1}\right)\left(-1+\frac{1-y_{k}}{f_{k}\left(y_{1}\right)}\right)$.

Since $f_{k}$ is decreasing, replacing $y_{1}$ by $y_{k}$ inside the parentheses gives the following upper bound for (2.10):

$$
y_{k+2} \ldots y_{2 k-1} f_{k}\left(y_{1}\right)\left(-1+D_{1}\left(y_{k}\right)\right) \leq 0,
$$

where the inequality follows from the fact that $D_{1} \leq D_{2} \leq \ldots \leq D_{k}=1$. This means that

$$
\frac{\partial}{\partial y_{k+1}} \widetilde{H}_{k} \leq 0
$$

and thus changing $y_{k+1}$ into $y_{k}$ in (2.9) increases the total expression.

Continuing inductively, assume that we have already shown that changing $y_{k+j}$ $\mapsto y_{k}$ for $1 \leq j \leq i-1$ gives an upper bound for (2.9). The next case is then the coefficient of $y_{k+i}$, which is

$$
\begin{gathered}
\begin{array}{c}
y_{k+i+1} \ldots y_{2 k-1}\left(-f_{k}\left(y_{1}\right) \ldots f_{k}\left(y_{i}\right)+\left(1-y_{k}\right) f_{k}\left(y_{1}\right) \ldots f_{k}\left(y_{i-1}\right)\right. \\
\left.+\ldots+\left(1-y_{k}\right) y_{k}^{i-1}\right)
\end{array} \\
=y_{k+i+1} \ldots y_{2 k-1} f_{k}\left(y_{1}\right) \ldots f_{k}\left(y_{i}\right)\left(-1+\frac{1-y_{k}}{f_{k}\left(y_{i}\right)}+\frac{\left(1-y_{k}\right) y_{k}}{f_{k}\left(y_{i-1}\right) f_{k}\left(y_{i}\right)}\right. \\
\left.+\ldots+\frac{\left(1-y_{k}\right) y_{k}^{i-1}}{f_{k}\left(y_{1}\right) \ldots f_{k}\left(y_{i}\right)}\right) \\
\leq y_{k+i+1} \ldots y_{2 k-1} f_{k}\left(y_{1}\right) \ldots f_{k}\left(y_{i}\right)\left(-1+D_{i}\left(y_{k}\right)\right) \leq 0,
\end{gathered}
$$

where we used the fact that $f_{k}$ is decreasing in order to shift all denominator arguments to $y_{k}$. Thus the partial derivative with respect to $y_{k+i}$ is also nonpositive, so the substitution $y_{k+i} \mapsto y_{k}$ gives an upper bound. Finally, once all $y_{k+i}$ for $1 \leq i \leq k-1$ have been set to $y_{k}$, we reach the desired conclusion, as

$$
\begin{aligned}
& \widetilde{H}\left(y_{1}, \ldots, y_{k}, y_{k}, \ldots, y_{k}\right) \leq f_{k}\left(y_{1}\right) \ldots f_{k}\left(y_{k}\right)( \frac{1-y_{k}}{f_{k}\left(y_{k}\right)}+\frac{\left(1-y_{k}\right) y_{k}}{f_{k}\left(y_{k-1}\right) f_{k}\left(y_{k}\right)} \\
&\left.+\ldots+\frac{\left(1-y_{k}\right) y_{k}^{k-1}}{f_{k}\left(y_{1}\right) \ldots f_{k}\left(y_{k}\right)}-1\right) \\
& \leq f_{k}\left(y_{1}\right) \ldots f_{k}\left(y_{k}\right)\left(D_{k}\left(y_{k}\right)-1\right)=0 .
\end{aligned}
$$


Proof of Proposition 2.7. As before, we use an inductive argument that relies on the recurrence (2.3). By the inductive hypothesis we obtain

$$
\begin{aligned}
\rho_{n+k} \leq & \prod_{i=1}^{n-k+1} f_{k}\left(1-u_{i}\right)\left(u_{n+k} f_{k}\left(1-u_{n-k+2}\right) \ldots f_{k}\left(1-u_{n}\right)\right. \\
& \quad+u_{n+k-1}\left(1-u_{n+k}\right) f_{k}\left(1-u_{n-k+2}\right) \ldots f_{k}\left(1-u_{n-1}\right) \\
& \left.\quad+\ldots+u_{n+1}\left(1-u_{n+2}\right) \ldots\left(1-u_{n+k}\right)\right) \\
& =\prod_{i=1}^{n-k+1} f_{k}\left(1-u_{i}\right)\left(\widetilde{H}_{k}\left(y_{n-k+2}, \ldots, y_{n+k}\right)+f_{k}\left(1-u_{n-k+2}\right) \ldots f_{k}\left(1-u_{n+1}\right)\right) \\
\leq & \prod_{i=1}^{n+1} f_{k}\left(1-u_{i}\right),
\end{aligned}
$$

where the conclusion is due to Proposition 2.8 .

Corollary 2.9. Assume that $A_{1}, \ldots, A_{n}$ are independent events with probabilities $0 \leq u_{1} \leq u_{2} \leq \ldots \leq u_{n} \leq 1$. Then

$$
\mathbf{P}\left(\left\{A_{i}\right\}_{i=1}^{n} \text { has no k-gaps }\right) \leq \prod_{i=k}^{n} f_{k}\left(1-u_{i}\right) .
$$

Proof. This follows directly from Proposition 2.7 since the probabilities $u_{n}, u_{n-1}$, $\ldots, u_{1}$ are decreasing. This gives

$$
\begin{aligned}
\mathbf{P}\left(\left\{A_{i}\right\}_{i=1}^{n} \text { has no } k \text {-gaps }\right) & =\mathbf{P}\left(\left\{A_{n-i+1}\right\}_{i=1}^{n} \text { has no } k \text {-gaps }\right) \\
& \leq \prod_{i=1}^{n-k+1} f_{k}\left(1-u_{n-i+1}\right)=\prod_{i=k}^{n} f_{k}\left(1-u_{i}\right) .
\end{aligned}
$$

Proof of Theorem 2.1. The theorem statement combines Proposition2.2 and Corollary 2.9 .

\section{Logarithmic PROBABILITy estimates}

In this section we largely adopt Holroyd, Liggett, and Romik's notation from [16] and define

$$
g_{k}(z):=-\log f_{k}\left(e^{-z}\right),
$$

which will allow us to translate products of $f_{k}$ (as seen in the probability estimates from Section 2) into (exponent) sums involving $g_{k}$. We catalog a number of useful properties of $g_{k}$ that we will need throughout the rest of the paper. The $k=1$ and $k=2$ cases of Lemma 3.1 were proven and used by Gravner and Holroyd in 11, 14. with some slight misstatements in parts $(i v)$ and $(v)$, although it should be noted that several of their proofs used the explicit formulas for $f_{1}$ and $f_{2}$, whereas our proofs use only the defining properties of $f_{k}$ for any $k$.

We also introduce notation for asymptotic inequalities that is used in this section and throughout the remainder of the paper. We write $f(z) \ll g(z)$ if there exists a constant $c>0$ such that $f(z) \leq c g(z)$ for sufficiently small $z$. Furthermore, if the 
constant $c$ does not depend on certain local parameters (which will be specified in use), we write $f(z) \underset{\text { unif }}{\ll} g(z)$.

Lemma 3.1. For all $k \geq 1$, the following are true:

(i) The function $g_{k}$ is decreasing and convex.

(ii) The function $g_{k}$ is integrable on $\mathbb{R}_{\geq 0}$, and satisfies the explicit evaluation

$$
\int_{0}^{\infty} g_{k}(z) d z=\lambda_{k} .
$$

(iii) As $z \rightarrow \infty, g_{k}$ has the asymptotic behavior

$$
g_{k}(z) \sim e^{-k z} .
$$

(iv) As $z \rightarrow 0$,

(v) As $z \rightarrow 0$,

$$
g_{k}(z) \sim \frac{1}{k} \log z^{-1}
$$

$$
g_{k}^{\prime}(z) \sim-\frac{1}{k z} .
$$

Proof. (i) This follows from Lemma 15 of [16].

(ii) This was proven as Theorem 1 of [16, and a second shorter proof was later given in [5].

(iii) Differentiate the recursion (2.1) to obtain

$$
\left(k f_{k}^{k-1}-(k+1) f_{k}^{k}\right) f_{k}^{\prime}=k x^{k-1}-(k+1) x^{k} .
$$

The boundary value $f_{k}(0)=1$ implies that $f_{k}^{\prime}(0)=0$. Repeatedly differentiating (3.1) then iteratively shows that

$$
f_{k}^{\prime \prime}(0)=\ldots=f_{k}^{(k-1)}(0)=0 .
$$

Finally, the $k$-th derivative yields a non-zero term, which is

$$
f_{k}^{(k)}(0)=-k !
$$

Therefore the Taylor expansion of $f_{k}$ around 0 has the form

$$
f_{k}(x)=1-x^{k}+O\left(x^{k+1}\right) .
$$

This leads to the stated assertion, since

$$
g_{k}(z)=-\log f_{k}\left(e^{-z}\right) \sim-\log \left(1-e^{-k z}\right) \sim e^{-k z}
$$

as $z \rightarrow \infty$.

(iv) Taking the logarithm of (2.1) yields

$$
k \log f_{k}(x)+\log \left(1-f_{k}(x)\right)=k \log x+\log (1-x) .
$$

Isolating $-\log f_{k}\left(e^{-z}\right)=g_{k}(z)$, we then apply the limiting value $f_{k}(1)=$ 0 to find that

$$
\begin{aligned}
\lim _{z \rightarrow 0} \frac{g_{k}\left(e^{-z}\right)}{\log \left(z^{-1}\right)} & =\lim _{z \rightarrow 0} \frac{\frac{1}{k}\left(\log \left(1-f_{k}\left(e^{-z}\right)\right)-k \log \left(e^{-z}\right)-\log \left(1-e^{-z}\right)\right)}{\log \left(z^{-1}\right)} \\
& =\lim _{z \rightarrow 0} \frac{\log \left(1-e^{-z}\right)}{k \log z}=\frac{1}{k} .
\end{aligned}
$$


(v) Equation (3.3) implies that

$$
-k g_{k}(z)+\log \left(1-f_{k}\left(e^{-z}\right)\right)=-k z+\log \left(1-e^{-z}\right) .
$$

Differentiating this equation gives

$$
-k g_{k}^{\prime}(z)+\frac{e^{-z} f_{k}^{\prime}\left(e^{-z}\right)}{1-f_{k}\left(e^{-z}\right)}=-k+\frac{e^{-z}}{1-e^{-z}} .
$$

We next claim that

$$
f_{k}(x) \sim(1-x)^{\frac{1}{k}} \quad \text { as } x \rightarrow 1 .
$$

This is enough to conclude $(v)$ Indeed, by (3.1) we have

$$
f_{k}^{\prime}(x)=\frac{k x^{k-1}-(k+1) x^{k}}{k f_{k}^{k-1}(x)-(k+1) f_{k}^{k}(x)} .
$$

As $z \rightarrow 0$ we then apply (3.5) to get

$$
f_{k}^{\prime}\left(e^{-z}\right) \ll \frac{1}{f^{k-1}\left(e^{-z}\right)} \ll \frac{1}{\left(1-e^{-z}\right)^{\frac{k-1}{k}}} \sim \frac{1}{z^{\frac{k-1}{k}}} .
$$

Therefore in (3.4) we have the asymptotic equality

$$
-k g_{k}^{\prime}(z)+f_{k}^{\prime}\left(e^{-z}\right) \sim-k+\frac{1}{z},
$$

yielding

$$
g_{k}^{\prime}(z) \sim-\frac{1}{k z} .
$$

We finish the proof by verifying (3.5). Taking the limit as $z \rightarrow 0$ in (3.3) and using the boundary value $f_{k}(1)=0$, we find that (again, $x=e^{-z}$ )

$$
k \log f_{k}(x) \sim \log (1-x),
$$

which gives (3.3).

\section{LOWER BOUND}

In this section we prove the lower bound in Theorem 1.1 for arbitrary $k$ by generalizing the combinatorial construction used in [11, and then using the new results from Section 3 to help estimate the corresponding probabilities. The general idea is to consider configurations that are sufficient for growth and that occur with large enough probability to give the tight lower bound. It should be noted that this construction also gives lower bounds for the original, non-localized $k$-percolation models, as any configuration with localized growth starting from the origin is clearly also sufficient for unrestricted growth (the same is not true of the upper bound in Section 5, as localized growth is not a necessary condition for unrestricted growth).

We first set some notation for rectangles in $\mathbb{Z}^{2}$. For a rectangle $R=\{a, \ldots, c\} \times$ $\{b, \ldots, d\}$, we denote its dimensions by

$$
\operatorname{dim}(R):=(c-a+1, d-b+1) .
$$

We also let $R(a, b)$ denote a rectangle with dimensions $(a, b)$ whose position may or may not be specified. Moreover, we visualize the base square at the origin as the lower-left corner of the northeast quadrant of the lattice $\mathbb{Z}^{2}$.

Following Gravner and Holroyd's basic argument in [11, we construct classes of configurations that always lead to indefinite growth. Figure 1 illustrates the two 
possibilities that we consider for the growth of a rectangle $R(a, a)$ to one of size $R(b, b)$. The first is "diagonal" growth, where $R(a, a)$ grows to $R(a+1, a+1)$, then to $R(a+2, a+2)$, and so on until $R(b, b)$ is active (with deviations from the diagonal of at most distance $k$ ). This sort of growth was shown to give the main (logarithmic) term of Theorem 1.1] in [14, 16]. The second sort of growth is horizontally "skew" growth, where growth proceeds first in the horizontal direction only, and then continues in the vertical direction only. The inclusion of the second growth event will be enough to increase the total probability by the claimed factor of $\exp \left(c_{1} s^{-1 / 2}\right)$.
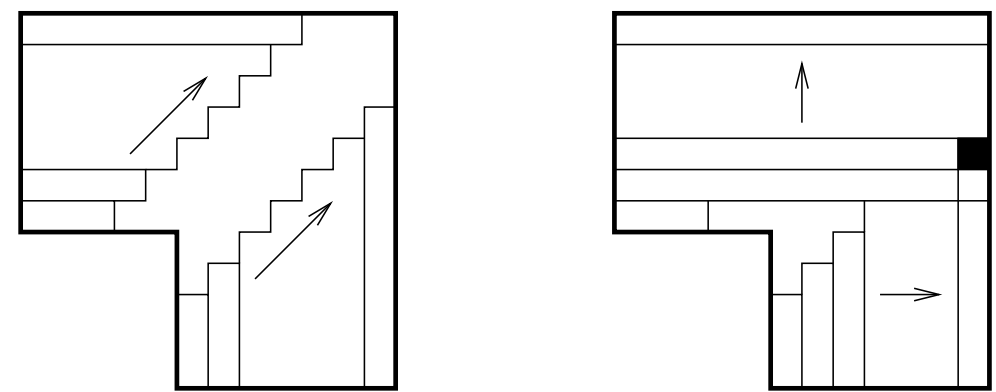

Figure 1. The columns and rows that define the events $\mathcal{D}_{k}(a, b)$ and $\mathcal{J}_{k}(a, b)$, respectively.

Definition 4.1. Consider the "stair-step" columns and rows defined such that for $i \geq k+1$, the column $\mathcal{C}_{i}$ at a distance $i$ to the right of the origin has height $i-k$, and the row $\mathcal{R}_{i}$ at height $i$ also has width $i-k$. If $b>a \geq k$, then the diagonal growth event $\mathcal{D}_{k}(a, b)$ is the event that the columns $\left\{\mathcal{C}_{a+1}, \ldots, \mathcal{C}_{b}\right\}$ and rows $\left\{\mathcal{R}_{a+1}, \ldots, \mathcal{R}_{b}\right\}$ have no $k$-gaps in the given configuration.

Definition 4.2. Suppose that $b-a \geq k+2$, let $\mathcal{C}_{a+i}$ have height $a+i-k$ for $1 \leq i \leq k$, and let all other $\mathcal{C}_{i}$ have height $a+1(a+k+1 \leq i \leq b)$. For the rows, let $\mathcal{R}_{a+1}$ have width $a-k+1$, let $\mathcal{R}_{i}$ have width $b-1$ for $a+2 \leq i \leq a+k+1$, and let $\mathcal{R}_{i}$ have width $b$ for $a+k+2 \leq i \leq b$. The (horizontally) skew event $\mathcal{J}_{k}(a, b)$ is the event where the following occur:

- $\mathcal{R}_{a+1}, \mathcal{C}_{a+1}, \mathcal{R}_{b}$ and $\mathcal{C}_{b}$ are non-empty,

- $\mathcal{R}_{a+2}, \ldots, \mathcal{R}_{a+k+1}$ are empty,

- the cell $(b, a+k+1)$ is occupied,

- $\left\{\mathcal{C}_{a+2}, \ldots, \mathcal{C}_{b-1}\right\}$ and $\left\{\mathcal{R}_{a+k+2}, \ldots, \mathcal{R}_{b-1}\right\}$ have no $k$-gaps.

It is clear that both events lead to further growth as stated in the following result.

Proposition 4.3. Suppose that we are given a configuration $\mathcal{C}$, and consider only those rectangles whose lower-left corner is at 0 .

(i) If $R(a, a)$ eventually becomes active and $\mathcal{D}_{k}(a, b)$ occurs, then $R(b-s, b-t)$ also becomes active for some $0 \leq s, t \leq k-1$. 
(ii) If $R(a-s, a-t)$ eventually becomes active for some $0 \leq s, t \leq k-1$ and $\mathcal{J}_{k}(a, b)$ occurs, then $R(b, b)$ also becomes active.

Definition 4.4. For $k \leq a_{1} \leq b_{1} \leq \ldots \leq a_{m} \leq b_{m} \leq L$ with $b_{i}-a_{i} \geq k+2$ for all $i$, define the growth event corresponding to these parameters as

$$
\begin{aligned}
& E_{k}\left(a_{1}, b_{1}, \ldots, a_{m}, b_{m}\right) \\
& \quad:=\mathcal{D}_{k}\left(k, a_{1}\right) \cap \bigcap_{i=1}^{m} \mathcal{J}_{k}\left(a_{i}, b_{i}\right) \cap \bigcap_{i=1}^{m-1} \mathcal{D}_{k}\left(b_{i}, a_{i+1}\right) \cap \mathcal{D}_{k}\left(b_{m}, L-1\right)
\end{aligned}
$$

$\bigcap\{(k \times k)$ lower left rectangle and cells $(1, L-1),(L-1,1)$ are occupied $\}$.

Lemma 4.5. Suppose that $\left\{a_{i}, b_{i}\right\}$ satisfy the conditions in Definition 4.4 .

(i) The various events appearing in the definition of a single occurrence of $E_{k}\left(a_{1}, \ldots, b_{m}\right)$ are independent.

(ii) If $E_{k}\left(a_{1}, \ldots, b_{m}\right)$ occurs, then $R(L)$ is eventually active.

(iii) For different choices of $a_{1}, \ldots, b_{m}$ the events $E_{k}\left(a_{1}, \ldots, b_{m}\right)$ are disjoint.

Proof. (i) Follows immediately from Definitions 4.1 and 4.2

(ii) Follows from Proposition 4.3

(iii) Let $\mathcal{R}_{n}, \mathcal{R}_{n+1}, \ldots, \mathcal{R}_{n+k-1}$ be the first $k$-gap among the stair-step rows. Then $\mathcal{R}_{n-1}$ is non-empty and begins a $\mathcal{J}_{k}$ event with $a_{1}=n-1$. Furthermore, the event ends with the first nonempty cell in the $(n+k-1)$-th row; the column position of this cell gives the value of $b_{1}$. Following this procedure iteratively uniquely determines all $a_{i}$ and $b_{i}$.

We next bound the probabilities of the events $\mathcal{D}_{k}$ and $\mathcal{J}_{k}$ in terms of the function $g_{k}$.

Lemma 4.6. The probability of the growth events satisfies the following lower bounds:

(i) If $b>a \geq k$, then

$$
\mathbf{P}\left(\mathcal{D}_{k}(a, b)\right) \geq \exp \left(-2 \sum_{i=a-(k-1)}^{b-k} g_{k}(i s)\right) .
$$

(ii) Let $c_{-}<c_{+}$be positive constants, $s \in\left(0, \frac{1}{2}\right)$, and $b \geq a+k+2$, with $a, b \in\left[c_{-} s^{-1}, c_{+} s^{-1}\right]$. Then

$$
\mathbf{P}\left(\mathcal{J}_{k}(a, b)\right) \underset{\text { unif }}{\gg} s \exp \left(g_{k}^{\prime}\left(c_{-}\right) s(b-a)^{2}-2 \sum_{i=a-(k-1)}^{b-k} g_{k}(i s)\right),
$$

where the asymptotic inequality is uniform over all $a, b$ in the given range.

Proof. (i) This follows directly from Proposition 2.2 and the definitions of $\mathcal{D}_{k}(a, b)$ and $g_{k}$. 
(ii) From the definition of $\mathcal{J}_{k}(a, b)$ and Proposition 2.2 we obtain

$$
\begin{gathered}
\mathbf{P}\left(\mathcal{J}_{k}(a, b)\right) \geq q^{k(b-1)}(1-q)\left(1-q^{a-k+1}\right)^{2}\left(1-q^{a+1}\right)\left(1-q^{b}\right) \\
\times \exp \left(-g_{k}((a-k+2) s)-\ldots-g_{k}(a s)-(b-a-k-1) g_{k}((a+1) s)\right. \\
\left.-(b-a-k-2) g_{k}(b s)\right)
\end{gathered}
$$

where the first $q$-power is for empty rows, the next several factors are for occupied rows and columns, and the final exponential terms are for the gap conditions among the remaining rows and columns. In the given ranges of $a, b$, the powers $q^{a}$ and $q^{b}$ may be treated as (uniform) asymptotic constants, as can the single terms $g_{k}((a-k+i) s)$ (of which there are $k-2)$. Also, $1-q$ is asymptotically $s$, so the overall bound becomes

$$
\begin{aligned}
& \mathbf{P}\left(\mathcal{J}_{k}(a, b)\right) \\
& \underset{\text { unif }}{\gg} s \exp \left(-(b-a)\left(g_{k}((a+1) s)+g_{k}(b s)\right)+(k+1) g_{k}((a+1) s)+(k+2) g_{k}(b s)\right) \\
& \geq s \exp \left(-(b-a)\left(g_{k}(a s)+g_{k}(b s)\right)\right) \text {. }
\end{aligned}
$$

The second inequality holds since the function $g_{k}$ is decreasing. It is also true that

$$
\exp \left(-\sum_{i=a-(k-1)}^{b-k} g_{k}(i s)\right) \leq \exp \left(-(b-a) g_{k}(b s)\right)
$$

again since $g_{k}$ is decreasing. Using this in (4.1), we have

$$
\begin{aligned}
\mathbf{P}\left(\mathcal{J}_{k}(a, b)\right) & \underset{\text { unif }}{\gg} s \exp \left(-(b-a)\left(g_{k}(a s)-g_{k}(b s)\right)-2 \sum_{i=a-(k-1)}^{b-k} g_{k}(i s)\right) \\
& \geq s \exp \left((b-a) g_{k}^{\prime}\left(c_{-}\right)(b s-a s)-2 \sum_{i=a-(k-1)}^{b-k} g_{k}(i s)\right)
\end{aligned}
$$

where we have used the convexity of $g_{k}$ for the final approximation.

With the combinatorial preliminaries finished, we now prove the lower bound by selecting a "window" of size proportional to $s^{-1}$.

Proof of lower bound in Theorem 1.1. Let $m:=\left\lfloor s^{-\frac{1}{2}} M\right\rfloor$, where $M<1$ is a positive constant that will be chosen later, and suppose that we have a sequence of parameters that satisfy

$$
s^{-1}<a_{1} \leq b_{1} \leq \ldots \leq a_{m} \leq b_{m}<\left\lfloor 2 s^{-1}\right\rfloor=: L
$$

with $b_{i}-a_{i} \in\left[k+2, s^{-\frac{1}{2}}\right]$ for all $i$. Lemma 4.6 shows that there is a constant $c>0$ such that we have the following lower bound for the probability of a growth 
event (note that the asymptotic bounds are uniform across all $a_{i}, b_{i}$ ):

$$
\begin{aligned}
& \mathbf{P}\left(E_{k}\left(a_{1}, \ldots, b_{m}\right)\right) \\
&= \mathbf{P}\left(\mathcal{D}_{k}\left(k, a_{1}\right)\right) \prod_{i=1}^{m} \mathbf{P}\left(\mathcal{J}_{k}\left(a_{i}, b_{i}\right)\right) \prod_{i=1}^{m-1} \mathbf{P}\left(\mathcal{D}_{k}\left(b_{i}, a_{i+1}\right)\right) \\
& \times \mathbf{P}\left(\mathcal{D}_{k}\left(b_{m}, L\right)\right) \mathbf{P}\left(k^{2}+2 \text { active sites }\right) \\
& \gg s_{\text {unif }}^{k^{2}+2} \exp \left(-2 \sum_{i=1}^{L-k} g_{k}(i s)\right) s^{m} c^{m} \exp \left(-c s \sum_{i=1}^{m}\left(b_{i}-a_{i}\right)^{2}\right) \\
& \underset{\text { unif }}{\gg} s^{m} c^{m} \exp \left(-2 \sum_{i=1}^{L-k} g_{k}(i s)-\left(k^{2}+2\right) \log s^{-1}\right),
\end{aligned}
$$

where for the last estimate we absorbed the last factor into the constant power $c^{m}$ by using the fact that $b_{i}-a_{i} \in\left[k+2, s^{-\frac{1}{2}}\right]$. The number of possible sequences $\left\{a_{i}, b_{i}\right\}$ is at least

$$
\begin{aligned}
\left(\begin{array}{c}
\left\lfloor s^{-1}-m s^{-\frac{1}{2}}\right\rfloor \\
m
\end{array}\right) & \left(s^{-\frac{1}{2}}-(k+2)\right)^{m} \gg\left(\begin{array}{c}
\left\lfloor s^{-1}(1-M)\right\rfloor \\
m
\end{array}\right)\left(s^{-\frac{1}{2}}\right)^{m} \\
& \gg\left(s^{-1}(1-M)\right)^{m}\left(\frac{s^{-\frac{1}{2}}}{m}\right)^{m} \gg s^{-m}\left(\frac{1-M}{M}\right)^{m},
\end{aligned}
$$

where the second approximation comes from Stirling's formula.

Finally, note that by Lemma 4.5 the event $E_{k}\left(a_{1}, \ldots, b_{m}\right)$ only guarantees growth out to $R(L, L)$. In order to achieve indefinite growth, we add the event $\mathcal{D}_{k}(L, \infty)$ as well, which means (in a slight abuse of notation) that there are no $k$-gaps in $\left\{\mathcal{C}_{L+1}, \mathcal{C}_{L+2}, \ldots\right\}$ and $\left\{\mathcal{R}_{L+1}, \mathcal{R}_{L+2}, \ldots\right\}$. This means that the entire northeast quadrant will become active, and we achieve indefinite growth in the whole plane by restricting further to the probability 1 condition that there are no empty semiinfinite lines in $\mathbb{Z}^{2}$ (this same argument was used by Gravner and Holroyd).

Combining (4.2) and (4.3), we obtain the estimate

$$
\begin{aligned}
\mathbf{P} \text { (indefinite growth) } & \geq \sum_{\text {sequences }\left\{a_{i}, b_{i}\right\}} \mathbf{P}\left(E_{k}\left(a_{1}, \ldots, b_{m}\right)\right) \cdot \mathbf{P}\left(\mathcal{D}_{k}(L, \infty)\right) \\
& \gg s^{-m}\left(\frac{1-M}{M}\right)^{m} s^{m} c^{m} \exp \left(-2 \lambda_{k} s^{-1}-\left(k^{2}+2\right) \log s^{-1}\right) \\
& \gg\left(c \cdot \frac{1-M}{M}\right)^{m} \exp \left(-2 \lambda_{k} s^{-1}-\left(k^{2}+2\right) \log s^{-1}\right) .
\end{aligned}
$$

Choosing $M$ sufficiently small so that $c \cdot \frac{1-M}{M}>1$, we obtain the lower bound

$$
\exp \left(-2 \lambda_{k} s^{-1}+c s^{-\frac{1}{2}}\right)
$$

which completes the proof.

\section{LOCAL UPPER BOUND}

We now turn to the upper bound in Theorem 1.1. Part of our proof follows Gravner and Holroyd's approach to the cases $k=1$ and $k=2$, and we improve 
several of their choices of parameters in order to achieve a tighter second-order term. We proceed through the technical preliminaries with unspecified parameters in order to show that our final choices are optimal for this approach. Our more significant contribution is a new combinatorial characterization of necessary growth conditions that allows us to adapt Gravner and Holroyd's scaling arguments to the case of general $k$.

We introduce "rectangle growth sequences" in order to encode the row and column conditions that occur in growing configurations. These sequences naturally contain a generalization of Gravner and Holroyd's "good sequences" from [11. Using some intricate combinatorial arguments, the probability of such a subsequence can be bounded, as can the total possible number of subsequences, and the combination of these estimates leads to the overall upper bounds.

For $k=1$, some of the arguments in Sections 5.1- 5.3 only apply to the modified model, and we explain the minor changes that are necessary for the $k=1$ Froböse model in Section 5.4

5.1. Preliminary combinatorial setup. We begin with the unspecified parameters. There are several important rough asymptotic properties that we will need for these parameters, so we define and list them now.

Definition 5.1. The parameters $A$ (lower dimension), $B$ (upper dimension), and $D$ (growth ratio) are assumed to be positive values that satisfy the following limiting relations as $s \rightarrow 0$ :

\begin{tabular}{|l||c|c|c|}
\hline Parameter name & $A$ & $B$ & $D$ \\
\hline Limiting value & $\infty$ & $\infty$ & 0 \\
\hline Asymptotics & $\log (A s) \sim \log s$ & $\log \left(A^{-1} B\right) \ll \log s^{-1}$ & $B D \gg 0$ \\
\hline Inequalities & $A<B / 2$ & $B \geq s^{-1} \log s^{-1}$ & $D<1$ \\
\hline
\end{tabular}

Next, we define the new combinatorial structure that we will use to encode and approximate the spread of active sites in the $k$-percolation model.

Definition 5.2. A rectangle growth sequence for an initial configuration $\mathcal{C}$ on $\mathbb{Z}^{2}$ is denoted by $\mathcal{S}(\mathcal{C})$, and is defined to be a sequence of rectangles

$$
\mathcal{S}(\mathcal{C}):=\left\{0=R_{1}^{\prime} \subsetneq \ldots \subsetneq R_{m}^{\prime} \subsetneq \ldots\right\}
$$

such that

(i) $S(\mathcal{C})$ is empty if the origin is not active in $\mathcal{C}$, and otherwise $R_{1}^{\prime}=\{0\}$.

(ii) Each $R_{i}^{\prime}$ has no $k$-gaps; in other words, there are no $k$ consecutive empty rows or columns in $R_{i}^{\prime}$.

(iii) $R_{i+1}^{\prime} \backslash R_{i}^{\prime}$ is contained in $\operatorname{Shell}\left(R_{i}^{\prime}\right)$, where $\operatorname{Shell}(R)$ is defined to be the width 1 boundary around any rectangle $R$.

Note that there may be many possible choices for the sequence $\mathcal{S}(\mathcal{C})$ depending on $\mathcal{C}$, and furthermore, that the sequence may be either finite or infinite. However, we are primarily interested in the class of maximal growth sequences in the current study, as we need to encode the fact that the percolation process proceeds so long as any growth is possible. Here maximality is defined in terms of the 
partial ordering of growth sequences given by (rectangle) containment, and it is straightforward to see that there is a well-defined "join" operation: if $R_{1}^{\prime}, R_{2}^{\prime}, \ldots$ and $S_{1}^{\prime}, S_{2}^{\prime}, \ldots$ are both growth sequences, then both are contained in the growth sequence $\left\langle R_{1}^{\prime}, S_{1}^{\prime}\right\rangle,\left\langle R_{2}^{\prime}, S_{2}^{\prime}\right\rangle, \ldots$, where for two rectangles $R$ and $S,\langle R, S\rangle$ denotes the span of $R$ and $S$ (the smallest rectangle that contains both $R$ and $S$ ).

Definition 5.3. A good configuration is an initial state on $\mathbb{Z}^{2}$ such that any maximal rectangle growth sequence is infinite.

This concept is useful for proving upper bounds for growth, as the next lemma shows that good configurations are a characterizing property of indefinite growth.

Lemma 5.4. If $\mathcal{C}$ has indefinite growth, then $\mathcal{C}$ is a good configuration.

Proof. We argue by contradiction and suppose that $\mathcal{C}$ is not a good configuration, and thus has a finite maximal growth sequence $\mathcal{S}(\mathcal{C})=\left\{0=R_{1}^{\prime} \subsetneq \ldots \subsetneq R_{n}^{\prime}\right\}$. By definition, such a sequence ends with a rectangle $R_{n}^{\prime}$ that is empty in its $k-1$ outermost rows and columns. Furthermore, $\operatorname{Shell}\left(R_{n}^{\prime}\right)$ must also be empty in $\mathcal{C}$. Thus $U:=R_{n}^{\prime} \cup \operatorname{Shell}\left(R_{n}^{\prime}\right)$ is empty in its $k$ outermost rows and columns, so that $R_{n-k+1}^{\prime}$ is the last rectangle in $\mathcal{S}$ that has occupied sites on its boundary.

According to the growth rules, there cannot be any active squares outside of $\mathcal{S}$ until there is first an active square somewhere in the $k$ outermost rows and columns of $U$. However, since these rows and columns are completely empty, they will remain so even if $R_{n-k+1}^{\prime}$ becomes completely active. This contradicts the assumption that $\mathcal{C}$ has indefinite growth, thus completing the proof.

Because of Lemma 5.4. we can use good configurations as an upper bound for indefinite growth, and we spend much of the remainder of this section showing that the arguments in [10] can still be applied to these configurations. We further classify good configurations into two types of behavior that, much like the events $\mathcal{J}_{k}$ and $\mathcal{D}_{k}$ in Section 4 . (roughly) correspond to whether the growth is "skew" or "diagonal".

Definition 5.5. A growth sequence escapes if there is an $R_{i}^{\prime}$ with dimensions $\left(a^{\prime}, b^{\prime}\right)$ such that $a^{\prime} \in[B, B+1]$ and $b^{\prime} \leq A$, or such that $a^{\prime} \leq A$ and $b^{\prime} \in[B, B+1]$.

We can now generalize Gravner and Holroyd's concept of a "good sequence" by considering appropriate subsequences of a growth sequence.

Definition 5.6. A good sequence is a sequence of rectangles $0 \in R_{1} \subsetneq \ldots \subsetneq R_{n+1}$ that satisfies the following conditions on the dimensions $\operatorname{dim} R_{i}=\left(a_{i}, b_{i}\right)$ :

(i) $\min \left\{a_{1}, b_{1}\right\} \in[A, A+1]$.

(ii) $a_{n}+b_{n} \leq B$.

(iii) $a_{n+1}+b_{n+1}>B$.

(iv) For $i=1, \ldots, n$ we have $s_{i} \geq a_{i} D$ or $t_{i} \geq b_{i} D$, where $s_{i}:=a_{i+1}-a_{i}$ and $t_{i}:=b_{i+1}-b_{i}$ are the successive dimension differences.

(v) For $i=1, \ldots, n$ we have $s_{i}<a_{i} D+2$ and $t_{i}<b_{i} D+2$.

For a rectangle $R$ define next the event

$$
G(R):=\{R \text { has no } k \text {-gaps in columns or rows }\} .
$$

Furthermore for two rectangles $R \subseteq R^{\prime}$, we define the subrectangles $S_{1}, \ldots, S_{8}$ (some of which may be empty) as in Figure 2 . 


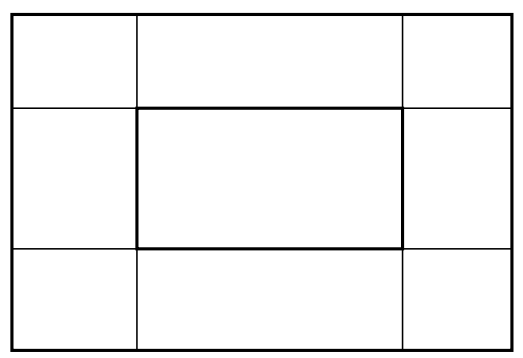

FiguRE 2. The rectangular regions defined by $R$ and $R^{\prime}$.

Definition 5.7. Let $D\left(R, R^{\prime}\right)$ denote the event that each of the two rectangles $S_{1} \cup S_{8} \cup S_{7}$ and $S_{3} \cup S_{4} \cup S_{5}$ have no $k$-gaps along the columns, and that each of the two rectangles $S_{1} \cup S_{2} \cup S_{3}$ and $S_{7} \cup S_{6} \cup S_{5}$ has no $k$-gaps along the rows.

Remark. One easily sees that $D\left(R, R^{\prime}\right)$ is necessary for the growth to proceed from $R$ to $R^{\prime}$.

Lemma 5.8. A good configuration $\mathcal{C}$ either has a good sequence $R_{1} \subsetneq \ldots \subsetneq R_{n+1}$ such that $G\left(R_{1}\right)$ and $\bigcap_{i=1}^{n} D\left(R_{i}, R_{i+1}\right)$ occur, or $S(\mathcal{C})$ escapes.

Proof. If $S(\mathcal{C})$ does not escape, then $R_{1} \subsetneq \ldots \subsetneq R_{n+1}$ can be taken as a subsequence of $S(\mathcal{C})$ that is determined solely by the rectangle dimensions. All of the conditions in Definition 5.6 are easily seen to be satisfied.

5.2. Probability estimates. We now approximate the probability of various events involving good sequences and follow the technical framework used in [11, 10, again using our new combinatorial definitions to unify the results for all $k$. Most of the proofs are straightforward generalizations of Gravner and Holroyd's, using our general estimates from Section 3 but we include all technical steps in order to be precise with certain " $k$-shifts" that occur.

We begin with a lemma that was proven in [16], which is also the special case where all probabilities are equal in the upper bound of our Proposition 2.7.

Lemma 5.9. If $R=R(a, b)$ is a rectangle, then

$$
\begin{aligned}
& \mathbf{P}(R \text { has no k-gaps in its columns or rows }) \\
& \quad \leq \begin{cases}\exp \left(-(a-(k-1)) g_{k}(b s)\right) & \text { if } a \leq b, \\
\exp \left(-(b-(k-1)) g_{k}(a s)\right) & \text { if } a \geq b .\end{cases}
\end{aligned}
$$

Next we prove $k$-analogs of several results in [10, beginning with the probability that the growth sequence $\mathcal{S}(\mathcal{C})$ escapes.

Lemma 5.10. If $s$ is sufficiently small, then there exists a constant $c>0$ such that

$$
\mathbf{P}(S(\mathcal{C}) \text { escapes }) \leq \exp \left(-c B \log s^{-1}\right)
$$


Proof. The number of possible rectangles $R$ such that the growth sequence escapes is at most $4 A^{2}(B+1)$. For such an $R$ Lemma 5.9 implies that as $s \rightarrow 0$,

$$
\mathbf{P}(G(R)) \leq \exp \left(-(B-(k-1)) g_{k}(A s)\right) \leq \exp \left(-c \cdot B g_{k}(A s)\right)
$$

for some constant $c$. We obtain this uniform bound by using the fact that $g_{k}$ is decreasing (Lemma 3.1 (i) ). By the assumptions in Definition [5.1, As $\rightarrow 0$ for $s \rightarrow 0$, so we may use Lemma 3.1 (iv) giving that

$$
\mathbf{P}(S(\mathcal{C}) \text { escapes }) \leq 4 A^{2}(B+1) \exp \left(-\frac{B c}{k} \log (A s)^{-1}\right) \leq \exp \left(-c B \log s^{-1}\right) .
$$

The final bound follows since we can absorb the leading factor into the exponential error term, and also from the asymptotic

$$
\log (A s)^{-1} \sim \log s^{-1} .
$$

Next come several bounds related to good sequences, beginning with a uniform bound for the initial rectangle $R_{1}$.

Lemma 5.11. Let $R_{1}, \ldots, R_{n+1}$ be a good sequence of rectangles and let $a_{0}=b_{0}=$ $A, s_{0}=a_{1}-a_{0}, t_{0}=b_{1}-b_{0}$. Then we have for some constant $c>0$

$$
\mathbf{P}\left(G\left(R_{1}\right)\right) \leq \exp \left(-s_{0} g_{k}\left(b_{0} s\right)-t_{0} g_{k}\left(a_{0} s\right)+c A^{-1} B\right) .
$$

Proof. We assume without loss of generality that $a_{1} \geq b_{1}$, so $b_{1} \in[A, A+1]$. By Lemma 5.9 we have

$$
\mathbf{P}\left(G\left(R_{1}\right)\right) \leq \exp \left(-\left(a_{1}-(k-1)\right) g_{k}\left(b_{1} s\right)\right) .
$$

Thus, noting that $t_{0} \leq 1$,

$$
\begin{aligned}
& \frac{\mathbf{P}\left(G\left(R_{1}\right)\right)}{\exp \left(-s_{0} g_{k}\left(b_{0} s\right)-t_{0} g_{k}\left(a_{0} s\right)\right)} \\
& \quad \leq \exp \left(-\left(a_{1}-(k-1)\right) g_{k}\left(b_{1} s\right)+s_{0} g_{k}(A s)+t_{0} g_{k}(A s)\right) \\
& \leq \exp \left(s_{0}\left(g_{k}(A s)-g_{k}\left(b_{1} s\right)\right)-(A-(k-1)) g_{k}\left(b_{1} s\right)+g_{k}(A s)\right) \\
& \quad \leq \exp \left(s_{0}\left(g_{k}(A s)-g_{k}\left(b_{1} s\right)\right)+g_{k}(A s)\right) .
\end{aligned}
$$

Since $g_{k}$ is convex and monotonically decreasing (Lemma 3.1 $[(i)]$,

$$
0 \leq g_{k}(A s)-g_{k}\left(b_{1} s\right) \ll-s g_{k}^{\prime}(A s) \ll A^{-1},
$$

where for the last estimate we used Lemma $3.1(v)$. Moreover, Lemma $3.1(i v)$ gives

$$
g_{k}(A s) \ll \frac{1}{k} \log s^{-1} .
$$

Thus (5.1) can be estimated against

$$
\exp \left(c s_{0} A^{-1}+c \log s^{-1}\right) \leq \exp \left(c B A^{-1}\right),
$$

where the final inequality follows from our assumption that $B A^{-1} \gg \log s^{-1}$. 
Lemma 5.12. If $R \subseteq R^{\prime}$ are two rectangles with dimensions $(a, b)$ and $(a+\ell, b+m)$, respectively, then

$$
\begin{aligned}
\mathbf{P}\left(D\left(R, R^{\prime}\right)\right) \leq \exp \left(-(m-2(k-1)) g_{k}(a s)\right. & -(\ell-2(k-1)) g_{k}(b s) \\
& \left.+\ell m s \exp \left(k\left(g_{k}(a s)+g_{k}(b s)\right)\right)\right) .
\end{aligned}
$$

Proof. We use the same notation as in Figure 2 and split the event according to the total number of occupied sites in the corners $S_{1} \cup S_{3} \cup S_{5} \cup S_{7}$. The probability that exactly $j$ (out of a possible number of $\ell m$ ) such corner sites are non-empty is

$$
\left(\begin{array}{c}
\ell m \\
j
\end{array}\right)(1-q)^{j} q^{\ell m-j}
$$

The presence of these occupied corner sites divides $S_{4}$ and $S_{8}$ into column strips $\mathcal{C}_{1}, \ldots, \mathcal{C}_{\alpha}$ for some $\alpha \leq j+2$, where the strip $\mathcal{C}_{i}$ has corresponding width $c_{i}$. Using Lemma 5.9 we obtain a bound for the event that $S_{4}$ and $S_{8}$ have no $k$-gaps; namely

$$
\begin{aligned}
\mathbf{P}\left(S_{4}\right. \text { and } & \left.S_{8} \text { have no } k \text { gaps }\right) \\
& \leq \exp \left(-\left(c_{1}-(k-1)\right) g_{k}(b s)-\ldots-\left(c_{\alpha}-(k-1)\right) g_{k}(b s)\right) \\
& =\exp \left(-\left(c_{1}+\ldots+c_{\alpha}-\alpha(k-1)\right) g_{k}(b s)\right) \\
& \leq \exp \left(-(\ell-j-(j+2)(k-1)) g_{k}(b s)\right) \\
& =\exp \left(-(\ell-k j-2(k-1)) g_{k}(b s)\right) .
\end{aligned}
$$

A similar argument applies to the rows in $S_{2}$ and $S_{6}$, and the column and row events are independent. Thus

$$
\begin{aligned}
\mathbf{P}\left(D\left(R, R^{\prime}\right)\right) \leq \sum_{j=0}^{\ell m}\left(\begin{array}{c}
\ell m \\
j
\end{array}\right)(1-q)^{j} q^{\ell m-j} \exp ( & -(\ell-k j-2(k-1)) g_{k}(b s) \\
& \left.-(m-k j-2(k-1)) g_{k}(a s)\right) .
\end{aligned}
$$

The approximations $q \leq 1$ and $1-q \leq s$ then imply that

$$
\begin{gathered}
\mathbf{P}\left(D\left(R, R^{\prime}\right)\right) \leq \exp \left(-(\ell-2(k-1)) g_{k}(b s)-(m-2(k-1)) g_{k}(a s)\right) \\
\times \sum_{j=0}^{\ell m}\left(\begin{array}{c}
\ell m \\
j
\end{array}\right) s^{j} \exp \left(k j\left(g_{k}(b s)+g_{k}(a s)\right)\right) \\
=\exp \left(-(\ell-2(k-1)) g_{k}(b s)-(m-2(k-1)) g_{k}(a s)\right) \\
\times\left(1+s \exp \left(k\left(g_{k}(b s)+g_{k}(a s)\right)\right)\right) \\
\leq \exp \left(-(\ell-2(k-1)) g_{k}(b s)-(m-2(k-1)) g_{k}(a s)\right. \\
+s \ell m \exp \left(k\left(g_{k}(a s)+g_{k}(b s)\right)\right),
\end{gathered}
$$

where for the last inequality we used the crude estimate $1+x \leq e^{x}$. 
Several of the prior papers on this subject have used a general variational result for probabilities involving convex functions [10, 14, and we bound the resulting error terms for $g_{k}$ and our particular parameters.

Lemma 5.13 (Lemma 7 of [10]). Suppose that $A$ and $B$ are positive integers satisfying $2 A<B$, and that $\left(a_{i}, b_{i}\right)_{i=1, \ldots, n+1}$ satisfy $a_{0}=b_{0}=A, s_{i}:=a_{i+1}-a_{i} \geq 0$, $t_{i}:=b_{i+1}-b_{i} \geq 0$, as well as the first three properties of Definition [5.6. For any $s>0$ and any positive, smooth, convex, decreasing function $g:(0, \infty) \rightarrow(0, \infty)$,

$$
\sum_{i=1}^{n}\left(s_{i} g\left(b_{i} s\right)+t_{i} g\left(a_{i} s\right)\right) \geq \frac{2}{s} \int_{A s}^{B s} g(t) d t-2 B g\left(\frac{B s}{2}\right) .
$$

Remark. The parameters in this statement are slightly shifted from those used by Gravner and Holroyd, but the proof is analogous.

Corollary 5.14. If $A$ and $B$ are as in Definition 5.1, then for s sufficiently small there exists a constant $c>0$ such that

$$
\begin{aligned}
\sum_{i=1}^{n}\left(s_{i} g_{k}\left(b_{i} s\right)+t_{i} g_{k}\left(a_{i} s\right)\right) \geq & 2 \lambda_{k} s^{-1} \\
& -c\left(A \log s^{-1}-s^{-1} \exp (-k B s)-B \exp (-k B s / 2)\right) .
\end{aligned}
$$

Proof. We first consider the integral in the bound of Lemma 5.13 and write

$$
\int_{A s}^{B s} g_{k}(z) d z=\int_{0}^{\infty} g_{k}(z) d z-\int_{0}^{A s} g_{k}(z) d z-\int_{B s}^{\infty} g_{k}(z) d z
$$

The first integral equals $\lambda_{k}$ by Lemma 3.1 (ii) Using Lemma 3.1 (iv) and our assumptions on $A$, the second integral can be estimated by

$$
c A s \log (A s)^{-1} \sim c A s \log s^{-1} .
$$

Lemma 3.1 (iii) and assumptions on $B$ imply that the third integral can be estimated by

$$
c \int_{B s}^{\infty} e^{-k z} d z \sim c e^{-k B s} .
$$

Finally, for the second term of Lemma 5.13 Lemma 3.1] (iii) again gives that as $s \rightarrow 0$,

$$
B g_{k}\left(\frac{B s}{2}\right) \ll B \exp \left(-\frac{k}{2} B s\right) .
$$

Next we consider bounds involving the dimensions of a good sequence.

Lemma 5.15. Let $n$ and $a_{i}, b_{i}(i=1, \ldots, n+1)$ be positive integers and denote the successive differences by $s_{i}:=a_{i+1}-a_{i} \geq 0$ and $t_{i}:=b_{i+1}-b_{i} \geq 0$ for $i=1, \ldots, n$. Further assume that the dimensions satisfy all of the properties of a good sequence. Then for $s \rightarrow 0$ the following bounds are satisfied:

(i) $n \ll D^{-1} \log s^{-1}$,

(ii) $\sum_{i=1}^{n} \frac{s_{i} t_{i}}{a_{i} b_{i}} \ll D \log s^{-1}$. 
Proof. $\quad(i)$ To bound $n$, we use (i), (ii), and (iv) from Definition 5.6

$$
(1+D)^{n-1} \leq \frac{a_{n}}{a_{n-1}} \frac{a_{n-1}}{a_{n-2}} \ldots \frac{a_{2}}{a_{1}} \frac{b_{n}}{b_{n-1}} \frac{b_{n-1}}{b_{n-2}} \ldots \frac{b_{2}}{b_{1}}=\frac{a_{n}}{a_{1}} \frac{b_{n}}{b_{1}} \ll \frac{B^{2}}{A^{2}} .
$$

Taking logarithms yields

$$
(n-1) \log (1+D) \ll \log \left(\frac{B}{A}\right) \ll \log s^{-1} .
$$

Thus

$$
n D \ll \log s^{-1},
$$

which gives the claim.

(ii) Simple uniform bounds and part $(i)$ show that

$$
\sum_{i=1}^{n} \frac{s_{i} t_{i}}{a_{i} b_{i}} \ll D^{2} n \ll D \log s^{-1} .
$$

The above results are enough to bound the probability of any good sequence, and we further estimate the total number of such sequences.

Lemma 5.16. The number of good sequences of rectangles is at most

$$
\exp \left(c D^{-1}\left(\log s^{-1}\right)^{2}\right)
$$

where $c>0$ is some constant.

Proof. First, there are at most $4 B \cdot(A+1) B$ choices for $R_{1}$. Next, given $R_{i}$ there are less than $(B D)^{4}$ choices for $R_{i+1}$. The length of the sequence is $n+1$, where $n$ is bounded by Lemma 5.15, and we have an overall bound of

$$
\ll(A+1) B^{2}(B D)^{4 n+4} \leq \exp (c n \log B) \leq \exp \left(c D^{-1}\left(\log s^{-1}\right)^{2}\right) .
$$

We end with one additional technical estimate.

Lemma 5.17. Suppose that $B$ satisfies the preceding assumptions. Then for the range $0 \leq a \leq B$, there is a uniform asymptotic bound

$$
e^{g_{k}(a s)} \underset{\text { unif }}{\ll}\left(\frac{B}{a}\right)^{\frac{1}{k}} \quad \text { as } s \rightarrow 0 .
$$

Proof. As $z \rightarrow 0$, Lemma 3.1 (iv) implies that

$$
e^{g_{k}(z)} \ll \exp \left(\frac{1}{k} \log z^{-1}\right)=\frac{1}{z^{\frac{1}{k}}},
$$

and as $z \rightarrow \infty$, Lemma 3.1 (iii) gives

$$
e^{g_{k}(z)} \ll \exp \left(e^{-k z}\right) \ll 1 .
$$

Recall that $B s \rightarrow \infty$ as $s \rightarrow 0$. By continuity and the above asymptotics, there is thus a sufficiently large $M$ and a constant $c$ such that if $z \leq M$, then

$$
e^{g_{k}(z)} \leq c\left(\frac{M}{z}\right)^{\frac{1}{k}} .
$$


5.3. Proof of the upper bound in Theorem 1.1. We are now ready to prove the main result of this section. By Lemma 5.8, we have

$$
\begin{aligned}
\mathbf{P}(\text { indefinite growth }) \leq & \mathbf{P}(\mathcal{S}(\mathcal{C}) \text { escapes }) \\
& +\sum_{\substack{\text { good sequences } \\
R_{1}, \ldots, R_{n+1}}} \mathbf{P}\left(G\left(R_{1}\right)\right) \prod_{i=1}^{n} \mathbf{P}\left(D\left(R_{i}, R_{i+1}\right)\right) .
\end{aligned}
$$

We note for future reference that we will use Lemma5.10 to estimate $\mathbf{P}(S(C)$ escapes).

For the good sequences term, Lemma 5.12 says that

$$
\begin{aligned}
& \mathbf{P}\left(D\left(R_{i}, R_{i+1}\right)\right) \\
& \underset{\text { unif }}{\ll} \exp \left(-\left(t_{i}-2(k-1)\right) g_{k}\left(a_{i} s\right)-\left(s_{i}-2(k-1)\right) g_{k}\left(b_{i} s\right)\right) \\
& \quad \times \exp \left(s_{i} t_{i} s \exp \left(k\left(g_{k}\left(a_{i} s\right)+g_{k}\left(b_{i} s\right)\right)\right)\right) .
\end{aligned}
$$

Applying the uniform bound from Lemma 5.17 gives

$$
\mathbf{P}\left(D\left(R_{i}, R_{i+1}\right)\right) \underset{\text { unif }}{\ll} \exp \left(-\left(t_{i} g_{k}\left(a_{i} s\right)+s_{i} g_{k}\left(b_{i} s\right)\right)\right)\left(\frac{B^{2}}{a_{i} b_{i}}\right)^{\frac{2(k-1)}{k}} \exp \left(s_{i} t_{i} s\left(\frac{B^{2}}{a_{i} b_{i}}\right)\right) .
$$

We also use Lemma 5.11 to bound $\mathbf{P}\left(G\left(R_{1}\right)\right)$ and Lemma 5.16 to bound the number of good sequences. Combined with (5.3), this gives the following upper bound for the second term in (5.2):

$$
\begin{aligned}
& \exp \left(c D^{-1}\left(\log s^{-1}\right)^{2}-s_{0} g_{k}\left(b_{0} s\right)-t_{0} g_{k}\left(a_{0} s\right)+c A^{-1} B\right) \\
& \times \prod_{i=1}^{n} \exp \left(-\left(t_{i} g_{k}\left(a_{i} s\right)+s_{i} g_{k}\left(b_{i} s\right)\right)\right)\left(\frac{B^{2}}{a_{i} b_{i}}\right)^{\frac{2(k-1)}{k}} \exp \left(s_{i} t_{i} s\left(\frac{B^{2}}{a_{i} b_{i}}\right)\right) \\
& \underset{\text { unif }}{\ll} \exp \left(-\sum_{i=0}^{n}\left(s_{i} g_{k}\left(b_{i} s\right)+t_{i} g_{k}\left(a_{i} s\right)\right)\right) \exp \left(c D^{-1}\left(\log s^{-1}\right)^{2}+c A^{-1} B\right)\left(\frac{B^{2}}{A^{2}}\right)^{\frac{2(k-1)}{k} n} \\
& \times \exp \left(B^{2} s \sum_{i=1}^{n} \frac{s_{i} t_{i}}{a_{i} b_{i}}\right) .
\end{aligned}
$$

Using Corollary 5.14, the first (multiplicative) term in (5.4) is bounded above by

$$
\exp \left(-\frac{2 \lambda_{k}}{s}+c A \log s^{-1}+c s^{-1} \exp (-k B s)\right) \exp \left(B c \exp \left(-\frac{k}{2} B s\right)\right) \text {. }
$$

Next, Lemma 5.15 bounds the third term as

$$
\left(\frac{B^{2}}{A^{2}}\right)^{c D^{-1} \log s^{-1}} \leq \exp \left(c D^{-1}\left(\log s^{-1}\right)^{2}\right) \text {. }
$$

Finally, the last term of (5.4) is also bounded by Lemma 5.15, giving

$$
\exp \left(B^{2} s \sum_{i=1}^{n} \frac{s_{i} t_{i}}{a_{i} b_{i}}\right) \leq \exp \left(B^{2} D s \log s^{-1}\right) .
$$


Combining all of these approximations gives the following upper bound for the second summand of (5.4):

$$
\begin{gathered}
\exp \left(-2 \lambda_{k} s^{-1}+c\left(A \log s^{-1}+A^{-1} B+D^{-1}\left(\log s^{-1}\right)^{2}+s B^{2} D \log s^{-1}\right)\right. \\
\left.+c\left(s^{-1} \exp (-k B s)+B \exp \left(-\frac{k B s}{2}\right)\right)\right) .
\end{gathered}
$$

By assumption $B \gg s^{-1} \log s^{-1}$, and thus the last two terms are simply part of the error. Examining the rest of the expression shows that this error is in fact optimized when $B \sim s^{-1} \log s^{-1}$. We now assume that we can write

$$
A \sim s^{-\alpha}\left(\log s^{-1}\right)^{\gamma} \quad D \sim s^{\beta}\left(\log s^{-1}\right)^{\delta}
$$

for some positive constants $\alpha, \beta$ and some constants $\gamma, \delta$, and tabulate the corresponding powers that arise from the relevant terms in (5.5):

\begin{tabular}{|l||c|c|c|c|}
\hline Term & $A \log s^{-1}$ & $A^{-1} B$ & $D^{-1}\left(\log s^{-1}\right)^{2}$ & $s B^{2} D \log s^{-1}$ \\
\hline$s$-power & $-\alpha$ & $-1+\alpha$ & $-\beta$ & $-1+\beta$ \\
\hline $\log s^{-1}$-power & $1+\gamma$ & $1-\gamma$ & $2-\delta$ & $3+\delta$ \\
\hline
\end{tabular}

We first consider the $s$-powers and easily see that the optimal choices are $\alpha=\beta=\frac{1}{2}$. Turning to the $\log s^{-1}$-powers, we find that the best choices are $\gamma=0, \delta=-\frac{1}{2}$, and that the second summand of (5.2) can be bounded by

$$
\exp \left(-2 \lambda_{k} s^{-1}+c s^{-\frac{1}{2}}\left(\log s^{-1}\right)^{\frac{5}{2}}\right) .
$$

Furthermore, recalling Lemma 5.10 and our discussion of the parameter $B$, we have the competing bound

$$
\mathbf{P}(\mathcal{S}(\mathcal{C}) \text { escapes }) \ll \exp \left(-c s^{-1}\left(\log s^{-1}\right)^{2}\right) .
$$

We can now prove the theorem statement as

$$
\begin{aligned}
\mathbf{P}(\text { indefinite growth }) \leq & \exp \left(-2 \lambda_{k} s^{-1}\right)\left(\exp \left(s^{-1}\left(2 \lambda_{k}-c\left(\log s^{-1}\right)^{2}\right)\right)\right. \\
& \left.+\exp \left(c s^{-\frac{1}{2}}\left(\log s^{-1}\right)^{\frac{5}{2}}\right)\right) \\
& \ll \exp \left(-2 \lambda_{k} s^{-1}+c s^{-\frac{1}{2}}\left(\log s^{-1}\right)^{\frac{5}{2}}\right) .
\end{aligned}
$$

5.4. Upper bound for the Froböse model. We end our study of percolation models with the Froböse model and prove the remaining $k=1$ case of Theorem 1.1 . It is only necessary to briefly mention the difference in comparison with the $k=1$ modified case. As before a growing configuration has a rectangle growth sequence, but in this case each shell not only has to be non-empty, but must also have noncorner occupied sites. Thus growth happens in only one direction at a time, which allows us to use disjointedness and the van der Berg-Kesten (BK) inequality rather 
than the corner decomposition of Lemma 5.12. To be more precise (using the same notation as before), if $R$ grows to $R^{\prime}$, then the disjoint intersection of events

$$
\left(\bigcap_{i=1}^{m} \mathcal{R}_{i} \text { non-empty }\right) \circ\left(\bigcap_{i=1}^{\ell} \mathcal{C}_{i} \text { non-empty }\right)
$$

occurs (here disjointedness means that it is possible to choose $m+\ell$ distinct nonempty cells, one for each column and row). The BK inequality [13] then implies that

$$
\mathbf{P}\left(D\left(R_{i}, R_{i+1}\right)\right) \leq\left(1-q^{b_{i}+t_{i}}\right)^{s_{i}}\left(1-q^{a_{i}+s_{i}}\right)^{t} .
$$

Therefore

$$
\begin{aligned}
& \sum_{\substack{\text { good sequences } \\
R_{1}, \ldots, R_{n+1}}} \mathbf{P}\left(G\left(R_{1}\right)\right) \prod_{i=1}^{n} \mathbf{P}\left(D\left(R_{i}, R_{i+1}\right)\right) \\
& \ll \exp \left(D^{-1}\left(\log s^{-1}\right)^{2}-2 s^{-1}\left(\lambda_{1}-A s \log s^{-1}-\exp (-B s)\right)\right. \\
& \left.+B \exp (-s B / 2)+A^{-1} B\right) \\
& \ll \exp \left(-2 \lambda_{1} s^{-1}+c\left(D^{-1}\left(\log s^{-1}\right)^{2}+A \log s^{-1}+A^{-1} B\right)\right. \\
& +c(s \exp (-B s)+B \exp (-B s / 2))) .
\end{aligned}
$$

As before we choose $B=s^{-1} \log s^{-1}$ and write $A=s^{-\alpha}\left(\log s^{-1}\right)^{\gamma}, D=s^{\beta}\left(\log s^{-1}\right)^{\delta}$. We see that the error is optimized for $\alpha=\frac{1}{2}, \beta=\frac{1}{2}, \gamma=0$, and $\delta=1$. This gives us a savings of $\log s^{-1}$ over Gravner and Holroyd's arguments.

\section{Proof of Theorem 1.3}

We next turn to the improved probability bound. Using Theorem 2.1 with $u_{i}=1-e^{-i s}$ yields

$$
\prod_{j=1}^{n} f_{k}\left(e^{-j s}\right) \leq \mathbf{P}\left(\left\{A_{j}\right\}_{j=1}^{n} \text { has no } k \text {-gaps }\right) \leq \prod_{j=k}^{n} f_{k}\left(e^{-j s}\right) .
$$

Since the events form a decreasing, nested sequence, we may take the limit as $n \rightarrow \infty$. This gives

$$
\begin{aligned}
\exp \left(-\sum_{j=1}^{\infty} g_{k}(j s)\right) & \leq \mathbf{P}\left(\left\{A_{i}\right\}_{j=1}^{\infty} \text { has no } k \text {-gaps }\right) \\
& \leq \exp \left(-\sum_{j=k}^{\infty} g_{k}(j s)\right) .
\end{aligned}
$$

We now use the Integral Comparison Theorem, which states that if $h(z)$ is a decreasing, convex function such that $\lim _{z \rightarrow \infty} h(z)=0$, then we have

$$
\frac{h(1)}{2}+\int_{1}^{\infty} h(z) d z \leq \sum_{j=1}^{\infty} h(j) \leq \int_{0}^{\infty} h(z) d z .
$$


This gives

$$
\begin{aligned}
\exp \left(-\int_{0}^{\infty} g_{k}(z s) d z\right) & \leq \mathbf{P}\left(\left\{A_{j}\right\}_{j=1}^{\infty} \text { has no } k \text {-gaps }\right) \\
& \leq \exp \left(-\frac{g_{k}(k s)}{2}-\int_{0}^{\infty} g_{k}(z s) d z+\int_{0}^{k} g_{k}(z s) d z\right) .
\end{aligned}
$$

Recall the integral evaluation from Lemma 3.1 (ii), and make the substitution $w=z s$. Then the lower bound in (6.2) is simply

$$
\mathbf{P}\left(\left\{A_{j}\right\}_{j=1}^{\infty} \text { has no } k \text {-gaps }\right) \geq \exp \left(-\int_{0}^{\infty} g_{k}(w) \frac{d w}{s}\right)=\exp \left(-\lambda_{k} s^{-1}\right) .
$$

The upper bound becomes

$$
\mathbf{P}\left(\left\{A_{j}\right\}_{j=1}^{\infty} \text { has no } k \text {-gaps }\right) \leq \exp \left(-\lambda_{k} s^{-1}-\frac{g_{k}(k s)}{2}+\frac{1}{s} \int_{0}^{k s} g_{k}(w) d w\right) .
$$

By Lemma $3.1(i v)$, this has the asymptotic behavior

$$
\begin{array}{r}
\mathbf{P}\left(\left\{A_{j}\right\}_{j=1}^{\infty} \text { has no } k \text {-gaps }\right) \leq \exp \left(-\lambda_{k} s^{-1}-\frac{1}{2 k}(1+o(1)) \log s^{-1}\right. \\
\left.+\log s^{-1}(1+o(1))\right)
\end{array}
$$

where the last term follows from the integral estimate

$$
\begin{aligned}
& \int_{0}^{k s} g_{k}(w) d w \leq \int_{0}^{k s}(1+o(1))\left(-\frac{1}{k} \log w\right) d w=\left(-\frac{1}{k}+o(1)\right)\left(w \log w-\left.w\right|_{0} ^{k s}\right) \\
& =\left(-\frac{1}{k}+o(1)\right)(k s \log (k s)-k s)=-s \log s(1+o(1)) .
\end{aligned}
$$

This gives

$$
\exp \left(-\lambda_{k} s^{-1}\right) \leq \mathbf{P}\left(\left\{A_{j}\right\}_{j=1}^{\infty} \text { has no } k \text {-gaps }\right) \leq s^{-\frac{(2 k-1)}{2 k}(1+o(1))} \cdot \exp \left(-\lambda_{k} s^{-1}\right),
$$

as claimed.

Remark. The upper and lower bounds in (6.1) are easily seen to differ by a factor of at most $s^{-(k-1) / k}$ in the asymptotic limit. By using the Integral Comparison Theorem to write the final bound in the form of (6.3), we have introduced the additional error factor of $s^{-1 / 2 k}$.

\section{ACKNOWLEDGMENTS}

The authors thank Michael Aizenman, Aernout van Enter, Alexander Holroyd, Rob Morris, and Dan Romik for helpful discussions regarding the history of bootstrap percolation, as well as for informing us of other recent developments in the subject. 


\section{REFERENCES}

[1] J. Adler, D. Stauffer, and A. Aharony, Comparison of bootstrap percolation models, J. Phys. (A) 22 (1989), L297-L301.

[2] M. Aizenman and J. Lebowitz, Metastability effects in bootstrap percolation, J. Phys. (A) 21 (1988), 3801-3813. MR968311 (90e:82047)

[3] G. Andrews, Partitions with short sequences and mock theta functions, Proc. Nat. Acad. Sci. 102 (2005), 4666-4671. MR2139704 (2006a:11131)

[4] G. Andrews, The theory of partitions, Cambridge University Press, Cambridge, 1998. MR1634067 (99c:11126)

[5] G. Andrews, H. Eriksson, F. Petrov, and D. Romik, Integrals, partitions and MacMahon's theorem, J. Comb. Theory (A) 114 (2007), 545-554. MR2310749(2008j:05035)

[6] J. Balogh, B. Bollobás, and R. Morris, Bootstrap percolation in high dimensions, Combin. Probab. Comput. 19 (2010), no. 5-6, 643-692. MR2726074

[7] J. Balogh, B. Bollobás, and R. Morris, Bootstrap percolation in three dimensions, Ann. Probab. 37 (2009), no. 4, 1329-1380. MR2546747(2011d:60278)

[8] K. Bringmann and K. Mahlburg, An extension of the Hardy-Ramanujan circle method and applications to partitions without sequences, to appear in Amer. J. of Math.

[9] K. Froböse, Finite-size effects in a cellular automaton for diffusion, J. Stat. Phys. 55 (1989), 1285-1292. MR 1002492 (90c:82004)

[10] J. Gravner and A. Holroyd, Local bootstrap percolation, Electronic Journal of Probability 14 (2009), 385-399. MR2480546 (2010c:60292)

[11] J. Gravner and A. Holroyd, Slow convergence in bootstrap percolation, The Annals of Applied Probability 18 (2008), 909-928. MR2418233 (2009d:60325)

[12] J. Gravner, A. Holroyd, and R. Morris, A sharper threshold for bootstrap percolation in two dimensions, to appear in Prob. Theory and Related Fields.

[13] G. Grimmett, Percolation, Springer-Verlag, second edition, 1999. MR.1707339 (2001a:60114)

[14] A. Holroyd, Sharp metastability threshold for two-dimensional bootstrap percolation, Probability Theory and Related Fields 125 (2003), 195-224. MR.1961342(2003k:60257)

[15] A. Holroyd, The metastability threshold for modified bootstrap percolation in d dimensions, Electronic Journal of Probability 11 (2006), 418-433. MR2223042 (2007a:82023)

[16] A. Holroyd, T. Liggett, and D. Romik, Integrals, partitions, and cellular automata, Trans. of the AMS 356 (2004), 3349-3368. MR2052953 (2005b:60018)

[17] R. Schonmann, On the behavior of some cellular automata related to bootstrap percolation, Ann. Prob. 20 (1992), 174-193. MR.1143417 (93b:60231)

[18] A. van Enter, Proof of Straley's argument for bootstrap percolation, J. Statist. Phys. 48 (1987), no. 3-4, 943-945. MR914911 (88j:82024)

[19] G. Watson, The final problem: An account of the mock theta functions, J. London Math. Soc. 11 (1936), 55-80. MR1862757

Mathematical Institute, University of Cologne, Weyertal 86-90, 50931 Cologne, Germany

E-mail address: kbringma@math.uni-koeln.de

Department of Mathematics, Princeton University, Princeton, New Jersey 08544

E-mail address: mahlburg@math.princeton.edu 